\title{
SINGULAR STOCHASTIC CONTROL AND OPTIMAL STOPPING WITH PARTIAL INFORMATION OF ITÔ-LÉVY PROCESSES*
}

\author{
BERNT ØKSENDAL ${ }^{\dagger}$ AND AGNÈS SULEM ${ }^{\ddagger}$
}

\begin{abstract}
We study partial information, possibly non-Markovian, singular stochastic control of Itô-Lévy processes and obtain general maximum principles. The results are used to find connections between singular stochastic control, reflected backward stochastic differential equations, and optimal stopping in the partial information case. As an application we give an explicit solution to a class of optimal stopping problems with finite horizon and partial information.
\end{abstract}

Key words. singular stochastic control, maximum principles, reflected BSDEs, optimal stopping, partial information, Itô-Lévy processes, jump diffusions

AMS subject classifications. 60H, 93E20, 60G51, 60H05

DOI. $10.1137 / 100793931$

1. Introduction. The aim of this paper is to establish stochastic maximum principles for partial information singular control problems of jump diffusions and to study relations with some associated reflected backward stochastic differential equations (RBSDEs) and optimal stopping problems.

To the best of our knowledge, the first paper which proves a maximum principle for singular control is Cadenillas and Haussmann [8], which deals with the case with no jumps and with full information. A connection between singular control and optimal stopping for Brownian motion was first established by Karatzas and Shreve [14] and generalized to geometric Brownian motion by Baldursson and Karatzas [5]. This was extended by Boetius and Kohlmann [7] and subsequently extended further by Benth and Reikvam [6] to more general continuous diffusions. More recently, maximum principles for singular stochastic control problems have been studied in $[1,2,3,4]$. None of these papers deal with jumps in the state dynamics and none of them deal with partial information control. Here we study general singular control problems of Itô ${ }^{-}$ Lévy processes, in which the controller has only partial information and the system is not necessarily Markovian. This allows for modeling of more general cases than before.

Singular control and optimal stopping are also related to impulse control. For example, an impulse control problem can be represented as a limit of iterated optimal stopping problems. See, e.g., [16, Chapter 7]. A maximum principle for linear forwardbackward systems involving impulse control can be found in [24].

We point out the difference between partial information and partial observation models. Concerning the latter, the information $\mathcal{E}_{t}$ available to the controller at time $\mathrm{t}$ is a noisy observation of the state (see, e.g., $[22,23,25]$ ). In such cases one can sometimes use filtering theory to transform the partial observation problem to a related

${ }^{*}$ Received by the editors May 3, 2010; accepted for publication (in revised form) July 2, 2012; published electronically August 16, 2012. The research leading to these results has received funding from the European Research Council under the European Community's Seventh Framework Programme (FP7/2007-2013)/ERC grant agreement 228087.

http://www.siam.org/journals/sicon/50-4/79393.html

${ }^{\dagger}$ Center of Mathematics for Applications, Department of Mathematics, University of Oslo, Blindern, N-0316 Oslo, Norway (oksendal@math.uio.no). This author was partially supported by the Institute for Mathematical Sciences, Singapore.

${ }^{\ddagger}$ INRIA Paris-Rocquencourt, Domaine de Voluceau, Rocquencourt, BP 105, Le Chesnay Cedex, 78153, France (agnes.sulem@inria.fr). 
problem with full information. The partial information problems considered in this paper, however, deal with the more general cases where we simply assume that the information flow $\mathcal{E}_{t}$ is a subfiltration of the full information $\mathcal{F}_{t}$.

Some partial information control problems can be reduced to partial observation problems and then solved by using filtering theory, but not all. For example, it seems to be difficult to handle the the situation with delayed information flow, i.e., $\mathcal{E}_{t}=\mathcal{F}_{t-\delta}$ with $\delta>0$ by using partial observation techniques.

The first part of the paper (section 2) is dedicated to the statement of stochastic maximum principles. Two different approaches are considered: (i) by using Malliavin calculus, leading to generalized variational inequalities for partial information singular control of possibly non-Markovian systems (subsection 2.2) and (ii) by introducing a singular control version of the Hamiltonian and using backward stochastic differential equations (BSDEs) for the adjoint processes to obtain partial information maximum principles for such problems (subsections 2.3 and 2.4). We show that the two methods are related, and we find a connection between them. In the second part of the paper (section 3), we study the relations between optimal singular control for jumps diffusions with partial information with general RBSDEs and optimal stopping. We first give a connection between the generalized variational inequalities found in section 2 and RBSDEs (subsection (3.1)). These are shown to be equivalent to general optimal stopping problems for such processes (subsections (3.2)). Combining this, a connection between singular control and optimal stopping is obtained in subsection 3.3. An illustrating example is provided in section 4. There we study a monotone-follower problem and arrive at an explicit solution of a class of optimal stopping problems with finite horizon and partial information. Indeed, it was one of the motivations of this paper to be able to handle partial information optimal stopping problems. This is a type of a problem which, it seems, has not been studied before.

\section{Maximum principles for optimal singular control.}

2.1. Formulation of the singular control problem. Consider a controlled singular Itô-Lévy process $X(t)=X^{\xi}(t)$ of the form $X\left(0^{-}\right)=x \in \mathbb{R}$ and

$$
\begin{aligned}
d X(t)= & b(t, X(t), \omega) d t+\sigma(t, X(t), \omega) d B(t) \\
& +\int_{\mathbb{R}_{0}} \theta\left(t, X\left(t^{-}\right), z, \omega\right) \tilde{N}(d t, d z)+\lambda(t, X(t), \omega) d \xi(t) ; t \in[0, T]
\end{aligned}
$$

defined on a probability space $\left(\Omega, \mathcal{F},\left(\mathcal{F}_{t}\right)_{t \geq 0}, P\right)$, where $t \rightarrow b(t, x), t \rightarrow \sigma(t, x)$, and $t \rightarrow \theta(t, x, z)$ are given $\mathcal{F}_{t}$-predictable processes for each $x \in \mathbb{R}, z \in \mathbb{R}_{0} \equiv \mathbb{R} \backslash\{0\}$. We assume that $b, \sigma, \theta$ and $\lambda$ are $C^{1}$ with respect to $x$ and that there exists $\epsilon>0$ such that

$$
\frac{\partial \theta}{\partial x}(t, x, z, \omega) \geq-1+\epsilon \quad \text { a.s. for all }(t, x, z) \in[0, T] \times \mathbb{R} \times \mathbb{R}_{0} .
$$

Here $\tilde{N}(d t, d z)$ is a compensated jump measure defined as $\tilde{N}(d t, d z)=N(d t, d z)-$ $\nu(d z) d t$, where $\nu$ is the Lévy measure of a Lévy process $\eta$ with jump measure, $N$ and $B$ is a Brownian motion (independent of $\tilde{N}$ ). We assume $E\left[\eta^{2}(t)\right]<\infty$ for all $t$, (i.e., $\left.\int_{\mathbb{R}_{0}} z^{2} \nu(d z)<\infty\right)$. Let

$$
\mathcal{E}_{t} \subseteq \mathcal{F}_{t} ; t \in[0, T]
$$

be a given subfiltration of $\mathcal{F}_{t}$ satisfying the usual assumptions. We assume that the process $t \rightarrow \lambda(t, x, \omega)$ is $\mathcal{E}_{t}$-adapted and continuous. 
Let $t \rightarrow f(t, x)$ and $t \rightarrow h(t, x)$ be given $\mathcal{F}_{t}$-predictable processes and $g(x)$ an $\mathcal{F}_{T}$-measurable random variable for each $x$. We assume that $f, g$, and $h$ are $C^{1}$ with respect to $x$. The process $\xi(t)=\xi(t, \omega)$ is our control process, assumed to be $\mathcal{E}_{t^{-}}$ adapted, càdlàg, and nondecreasing for each $\omega$ with $\xi\left(0^{-}\right)=0$. Moreover we require that $\xi$ is such that there exists a unique solution of $(2.1)$ and

$$
E\left[\int_{0}^{T}\|f(t, X(t), \omega)\| d t+\|g(X(T), \omega)\|+\int_{0}^{T}\left\|h\left(t, X\left(t^{-}\right), \omega\right)\right\| d \xi(t)\right]<+\infty .
$$

The set of such controls is denoted by $\mathcal{A}_{\mathcal{E}}$.

Since the case with classical control is well known, we choose in this paper to concentrate on the case with singular control only. However, by the same methods all the results could easily be extended to include a classical control in addition to the singular control.

Define the performance functional

$$
J(\xi)=E\left[\int_{0}^{T} f(t, X(t), \omega) d t+g(X(T), \omega)+\int_{0}^{T} h\left(t, X\left(t^{-}\right), \omega\right) d \xi(t)\right] .
$$

We want to find an optimal control $\xi^{*} \in \mathcal{A}_{\mathcal{E}}$ such that

$$
\Phi:=\sup _{\xi \in \mathcal{A}_{\mathcal{E}}} J(\xi)=J\left(\xi^{*}\right)
$$

For $\xi \in \mathcal{A}_{\mathcal{E}}$ we let $\mathcal{V}(\xi)$ denote the set of $\mathcal{E}_{t}$-adapted processes $\zeta$ of finite variation such that there exists $\delta=\delta(\xi)>0$ such that

$$
\xi+y \zeta \in \mathcal{A}_{\mathcal{E}} \text { for all } y \in[0, \delta] .
$$

For $\xi \in \mathcal{A}_{\mathcal{E}}$ and $\zeta \in \mathcal{V}(\xi)$ we have

$$
\begin{aligned}
\lim _{y \rightarrow 0^{+}} \frac{1}{y}(J(\xi+y \zeta)-J(\xi)) \\
=E\left[\int_{0}^{T} \frac{\partial f}{\partial x}(t, X(t)) \mathcal{Y}(t) d t+g^{\prime}(X(T)) \mathcal{Y}(T)\right. \\
\left.\quad+\int_{0}^{T} \frac{\partial h}{\partial x}\left(t, X\left(t^{-}\right)\right) \mathcal{Y}\left(t^{-}\right) d \xi(t)+\int_{0}^{T} h\left(t, X\left(t^{-}\right)\right) d \zeta(t)\right],
\end{aligned}
$$

where $\mathcal{Y}(t)$ is the derivative process defined by

$$
\mathcal{Y}(t)=\lim _{y \rightarrow 0^{+}} \frac{1}{y}\left(X^{\xi+y \zeta}(t)-X^{\xi}(t)\right) ; t \in[0, T] .
$$

Note that

$$
\mathcal{Y}(0)=\lim _{y \rightarrow 0^{+}} \frac{1}{y}\left(X^{\xi+y \zeta}(0)-X^{\xi}(0)\right)=\left.\frac{d}{d y} x\right|_{y=0}=0 .
$$

We have

$$
\begin{aligned}
d \mathcal{Y}(t)= & \mathcal{Y}\left(t^{-}\right)\left[\frac{\partial b}{\partial x}(t) d t+\frac{\partial \sigma}{\partial x}(t) d B(t)+\int_{\mathbb{R}_{0}} \frac{\partial \theta}{\partial x}(t, z) \tilde{N}(d t, d z)+\frac{\partial \lambda}{\partial x}(t) d \xi(t)\right] \\
& +\lambda(t, x) d \zeta(t),
\end{aligned}
$$

Copyright (c) by SIAM. Unauthorized reproduction of this article is prohibited. 
where we here (and in the following) are using the abbreviated notation

$$
\frac{\partial b}{\partial x}(t)=\frac{\partial b}{\partial x}(t, X(t)), \frac{\partial \sigma}{\partial x}(t)=\frac{\partial \sigma}{\partial x}(t, X(t)), \text { etc. }
$$

LEMma 2.1. The solution of (2.9) is

$$
\mathcal{Y}(t)=Z(t)\left[\int_{0}^{t} Z^{-1}\left(s^{-}\right) \lambda(s) d \zeta(s)+\sum_{0<s \leq t} Z^{-1}\left(s^{-}\right) \lambda(s) \alpha(s) \Delta \zeta(s)\right], \quad t \in[0, T],
$$

with $\Delta \zeta(s)=\zeta(s)-\zeta\left(s^{-}\right)$, where

$$
\alpha(s)=\frac{-\int_{\mathbb{R}_{0}} \frac{\partial \theta}{\partial x}(s, z) N(\{s\}, d z)-\frac{\partial \lambda}{\partial x}(t) \Delta \xi(t)}{1+\int_{\mathbb{R}_{0}} \frac{\partial \theta}{\partial x}(s, z) N(\{s\}, d z)+\frac{\partial \lambda}{\partial x}(t) \Delta \xi(t)}, s \in[0, T]
$$

and $Z(t)$ is the solution of the "homogeneous" version of (2.9), i.e., $Z(0)=1$ and

$$
d Z(t)=Z\left(t^{-}\right)\left[\frac{\partial b}{\partial x}(t) d t+\frac{\partial \sigma}{\partial x}(t) d B(t)+\int_{\mathbb{R}_{0}} \frac{\partial \theta}{\partial x}(t, z) \tilde{N}(d t, d z)+\frac{\partial \lambda}{\partial x}(t) d \xi(t)\right] .
$$

Proof. We try a solution $\mathcal{Y}(t)$ of the form $\mathcal{Y}(t)=Z(t) A(t)$, where

$$
A(t)=\int_{0}^{t} Z^{-1}\left(s^{-}\right) \lambda(s) d \zeta(s)+\beta(s)
$$

for some finite variation process $\beta(\cdot)$. By the Itô formula for semimartingales (see, e.g., [19, Theorem II.7.32]) we have

$$
d \mathcal{Y}(t)=Z\left(t^{-}\right) d A(t)+A\left(t^{-}\right) d Z(t)+d[Z, A]_{t},
$$

where

$$
\begin{aligned}
{[Z, A]_{t}=} & \sum_{0<s \leq t} \Delta Z(s) \Delta A(s) \\
= & \sum_{0<s \leq t} Z\left(s^{-}\right)\left[\int_{\mathbb{R}_{0}} \frac{\partial \theta}{\partial x}(s, z) N(\{s\}, d z)\right. \\
& \left.\quad+\frac{\partial \lambda}{\partial x}(s) \Delta \xi(s)\right]\left[Z^{-1}\left(s^{-}\right) \lambda(s) \Delta \zeta(s)+\Delta \beta(s)\right] \\
= & \sum_{0<s \leq t}\left[\int_{\mathbb{R}_{0}} \frac{\partial \theta}{\partial x}(s, z) N(\{s\}, d z)+\frac{\partial \lambda}{\partial x}(s) \Delta \xi(s)\right]\left[\lambda(s) \Delta \zeta(s)+Z\left(s^{-}\right) \Delta \beta(s)\right] .
\end{aligned}
$$

Copyright $@$ by SIAM. Unauthorized reproduction of this article is prohibited. 
Hence

$$
\begin{aligned}
d \mathcal{Y}(t)= & Z\left(t^{-}\right)\left[Z^{-1}\left(t^{-}\right) \lambda(t) d \zeta(t)+d \beta(t)\right] \\
& +\left[\int_{0}^{t} Z^{-1}\left(s^{-}\right) \lambda(s) d \zeta(s)+\beta(t)\right] Z\left(t^{-}\right) d \Gamma(t) \\
& +\left[\int_{\mathbb{R}_{0}} \frac{\partial \theta}{\partial x}(t, z) N(\{t\}, d z)+\frac{\partial \lambda}{\partial x}(t) \Delta \xi(t)\right]\left[\lambda(t) \Delta \zeta(t)+Z\left(t^{-}\right) \Delta \beta(t)\right] \\
= & \lambda(t) d \zeta(t)+\mathcal{Y}\left(t^{-}\right) d \Gamma(t) \\
& +Z\left(t^{-}\right) d \beta(t)+\left[\int_{\mathbb{R}_{0}} \frac{\partial \theta}{\partial x}(t, z) N(\{t\}, d z)\right. \\
& \left.+\frac{\partial \lambda}{\partial x}(t) \Delta \xi(t)\right]\left[\lambda(t) \Delta \zeta(t)+Z\left(t^{-}\right) \Delta \beta(t)\right]
\end{aligned}
$$

where

$$
d \Gamma(t)=\frac{\partial b}{\partial x}(t) d t+\frac{\partial \sigma}{\partial x}(t) d B(t)+\int_{\mathbb{R}_{0}} \frac{\partial \theta}{\partial x}(t, z) \tilde{N}(d t, d z)+\frac{\partial \lambda}{\partial x}(t) d \xi(t) .
$$

Thus (2.9) holds if we choose $\beta$ to be the pure jump càdlàg $\mathcal{F}_{t}$-adapted process given by

$$
\Delta \beta(t)=\frac{-\lambda(t) Z^{-1}\left(t^{-}\right)\left[\int_{\mathbb{R}_{0}} \frac{\partial \theta}{\partial x}(t, z) N(\{t\}, d z) \Delta \zeta(t)+\frac{\partial \lambda}{\partial x}(t) \Delta \xi(t)\right]}{1+\int_{\mathbb{R}_{0}} \frac{\partial \theta}{\partial x}(t, z) N(\{t\}, d z)+\frac{\partial \lambda}{\partial x}(t) \Delta \xi(t)}, t \in[0, T]
$$

Remark 2.2. Note that for any $F(s, z)$, we have

$$
\int_{\mathbb{R}_{0}} F(s, z) N(\{s\}, d z)=\left\{\begin{array}{l}
F(s, z) \text { if } \eta \text { has a jump of size } z \text { at } s \\
0 \text { otherwise. }
\end{array}\right.
$$

By the Itô formula we get that $Z$ is given by

$$
\begin{aligned}
Z(t)=\exp ( & \int_{0}^{t}\left\{\frac{\partial b}{\partial x}(r)-\frac{1}{2}\left(\frac{\partial \sigma}{\partial x}\right)^{2}(r)\right\} d r+\int_{0}^{t} \frac{\partial \lambda}{\partial x}(r) d \xi(r)+\int_{0}^{t} \frac{\partial \sigma}{\partial x}(r) d B(r) \\
& +\int_{0}^{t} \int_{\mathbb{R}_{0}} \ln \left(1+\frac{\partial \theta}{\partial x}(r, z)\right) \tilde{N}(d r, d z) \\
& \left.+\int_{0}^{t} \int_{\mathbb{R}_{0}}\left\{\ln \left(1+\frac{\partial \theta}{\partial x}(r, z)\right)-\frac{\partial \theta}{\partial x}(r, z)\right\} \nu(d z) d r\right) .
\end{aligned}
$$

In the following, we set

$$
G(t, s)=\frac{Z(s)}{Z(t)} \quad \text { for } t<s
$$

Copyright (c) by SIAM. Unauthorized reproduction of this article is prohibited. 
2.2. A Malliavin-calculus based maximum principle. In this section we use Malliavin calculus to get a stochastic maximum principle. This technique has been used earlier, e.g., in [15] and [17]. The main new ingredient here is the introduction of the singular control which requires special attention. In particular this control might be discontinuous, and it is necessary to distinguish between the jumps coming from the jump measure in the dynamics of $X$ and those from the controls and the perturbations.

Let $\mathbf{D}$ denote the space of random variables which are Malliavin-differentiable with respect both to Brownian motion $B$ and jump measure $N$. For $f \in \mathbf{D}$, let $D_{s} f$ denote the Malliavin derivative of $f$ at $s$ with respect to Brownian motion and $D_{s, z}$ denotes the Malliavin derivative of $f$ at $(s, z)$ with respect to the jump measure.

To study problem (2.4) we prove the following.

Lemma 2.3. Suppose $\xi \in \mathcal{A}_{\mathcal{E}}$ and $\zeta \in \mathcal{V}(\xi)$. Then

$$
\begin{aligned}
\lim _{y \rightarrow 0^{+}} \frac{1}{y}(J(\xi+y \zeta)-J(\xi)) \\
\quad=E\left[\int_{0}^{T}[\lambda(t) \tilde{p}(t)+h(t)] d \zeta^{c}(t)+\sum_{0<t \leq T}\{\lambda(t)(\tilde{p}(t)+S(t) \alpha(t))+h(t)\} \Delta \zeta(t)\right],
\end{aligned}
$$

where $\zeta^{c}(\cdot)$ denotes the continuous part of $\zeta(\cdot)$ and

$$
\begin{aligned}
S(t) & =\int_{t^{+}}^{T} G(t, s)\left[\frac{\partial H_{0}}{\partial x}(s) d s+R(s) \frac{\partial \lambda}{\partial x}(s) d \xi(s)\right] \\
\tilde{p}(t) & =R(t)+\int_{t}^{T} G(t, s)\left[\frac{\partial H_{0}}{\partial x}(s) d s+R(s) \frac{\partial \lambda}{\partial x}(s) d \xi(s)\right]=R(t)+S(t), \\
R(t) & =g^{\prime}(X(T))+\int_{t}^{T} \frac{\partial f}{\partial x}(s) d s+\int_{t^{+}}^{T} \frac{\partial h}{\partial x}(s) d \xi(s), \\
H_{0}(s, x) & =R(s) b(s, x)+D_{s} R(s) \sigma(s, x)+\int_{\mathbb{R}_{0}} D_{s, z} R(s) \theta(s, x, z) \nu(d z),
\end{aligned}
$$

provided that $R \in \mathbf{D}$.

Proof. For $\xi \in \mathcal{A}_{\mathcal{E}}$ and $\zeta \in \mathcal{V}(\xi)$, we compute the right-hand side (r.h.s.) of (2.6). Since $\mathcal{Y}(0)=0$, we have by the duality formulae for the Malliavin derivatives and integration by parts

$$
\begin{aligned}
& E\left[\int_{0}^{T} \frac{\partial f}{\partial x}(t) \mathcal{Y}(t) d t\right] \\
&=E\left[\int _ { 0 } ^ { T } \frac { \partial f } { \partial x } ( t ) \left(\int _ { 0 } ^ { t } \mathcal { Y } ( s ^ { - } ) \left[\frac{\partial b}{\partial x}(s) d s+\frac{\partial \sigma}{\partial x}(s) d B(s)+\int_{\mathbb{R}_{0}} \frac{\partial \theta}{\partial x}(s, z) \tilde{N}(d s, d z)\right.\right.\right. \\
&\left.\left.\left.+\frac{\partial \lambda}{\partial x}(s) d \xi(s)\right]+\lambda(s) d \zeta(s)\right) d t\right] \\
&=E\left[\int _ { 0 } ^ { T } \left(\int _ { 0 } ^ { t } \mathcal { Y } ( s ^ { - } ) \left\{\frac{\partial f}{\partial x}(t) \frac{\partial b}{\partial x}(s)+D_{s}\left(\frac{\partial f}{\partial x}(t)\right) \frac{\partial \sigma}{\partial x}(s)\right.\right.\right.
\end{aligned}
$$

Copyright $@$ by SIAM. Unauthorized reproduction of this article is prohibited. 


$$
\begin{gathered}
\left.+\int_{\mathbb{R}_{0}} D_{s, z}\left(\frac{\partial f}{\partial x}(t)\right) \frac{\partial \theta}{\partial x}(s, z) \nu(d z)\right\} d s \\
\left.\left.+\frac{\partial f}{\partial x}(t) \mathcal{Y}\left(s^{-}\right) \frac{\partial \lambda}{\partial x}(s) d \xi(s)+\frac{\partial f}{\partial x}(t) \lambda(s) d \zeta(s)\right) d t\right] \\
=E\left[\int _ { 0 } ^ { T } \left(\mathcal { Y } ( t ^ { - } ) \left\{\left(\int_{t}^{T} \frac{\partial f}{\partial x}(s) d s\right) \frac{\partial b}{\partial x}(t)+D_{t}\left(\int_{t}^{T} \frac{\partial f}{\partial x}(s) d s\right) \frac{\partial \sigma}{\partial x}(t)\right.\right.\right. \\
\left.+\int_{\mathbb{R}_{0}} D_{t, z}\left(\int_{t}^{T} \frac{\partial f}{\partial x}(s) d s\right) \frac{\partial \theta}{\partial x}(t, z) \nu(d z)\right\} d t \\
\left.\left.+\left(\int_{t}^{T} \frac{\partial f}{\partial x}(s) d s\right) \mathcal{Y}\left(t^{-}\right) \frac{\partial \lambda}{\partial x}(t) d \xi(t)+\left(\int_{t}^{T} \frac{\partial f}{\partial x}(s) d s\right) \lambda(t) d \zeta(t)\right)\right] .
\end{gathered}
$$

Similarly we get

$$
\begin{array}{r}
E\left[g^{\prime}(X(T)) \mathcal{Y}(T)\right]=E\left[\int _ { 0 } ^ { T } \left\{\mathcal { Y } ( t ^ { - } ) \left\{g^{\prime}(X(T)) \frac{\partial b}{\partial x}(t)+D_{t} g^{\prime}(X(T)) \frac{\partial \sigma}{\partial x}(t)\right.\right.\right. \\
\left.+\int_{\mathbb{R}_{0}} D_{t, z}\left(g^{\prime}(X(T))\right) \frac{\partial \theta}{\partial x}(t, z) \nu(d z)\right\} d t \\
\left.\left.+\mathcal{Y}\left(t^{-}\right) g^{\prime}(X(T)) \frac{\partial \lambda}{\partial x}(t) d \xi(t)+g^{\prime}(X(T)) \lambda(t) d \zeta(t)\right\}\right]
\end{array}
$$

and

$$
\begin{gathered}
E\left[\int_{0}^{T} \frac{\partial h}{\partial x}(t) \mathcal{Y}\left(t^{-}\right) d \xi(t)\right] \\
=E\left[\int _ { 0 } ^ { T } \left(\mathcal { Y } ( t ^ { - } ) \left\{\left(\int_{t^{+}}^{T} \frac{\partial h}{\partial x} d \xi(s)\right) \frac{\partial b}{\partial x}(t)+D_{t}\left(\int_{t^{+}}^{T} \frac{\partial h}{\partial x} d \xi(s)\right) \frac{\partial \sigma}{\partial x}(t)\right.\right.\right. \\
\left.\quad+\int_{\mathbb{R}_{0}} D_{t, z}\left(\int_{t^{+}}^{T} \frac{\partial h}{\partial x} d \xi(s)\right) \frac{\partial \theta}{\partial x}(t, z) \nu(d z)\right\} d t \\
\left.\left.+\left(\int_{t^{+}}^{T} \frac{\partial h}{\partial x} d \xi(s)\right) \mathcal{Y}\left(t^{-}\right) \frac{\partial \lambda}{\partial x}(t) d \xi(t)+\left(\int_{t^{+}}^{T} \frac{\partial h}{\partial x} d \xi(s)\right) \lambda(t) d \zeta(t)\right)\right] .
\end{gathered}
$$

Combining (2.6)-(2.22) and using the notation (2.18)-(2.19), we obtain

$$
\lim _{y \rightarrow 0^{+}} \frac{1}{y}(J(\xi+y \zeta)-J(\xi))=A_{1}(\zeta)+A_{2}(\zeta)
$$

where

$$
\begin{aligned}
& A_{1}(\zeta)=E\left[\int_{0}^{T} \mathcal{Y}\left(t^{-}\right)\left(\frac{\partial H_{0}}{\partial x}(t) d t+R(t) \frac{\partial \lambda}{\partial x}(t) d \xi(t)\right)\right] \\
& A_{2}(\zeta)=E\left[\int_{0}^{T}\{R(t) \lambda(t)+h(t)\} d \zeta(t)\right]
\end{aligned}
$$

Copyright (c) by SIAM. Unauthorized reproduction of this article is prohibited. 
This gives, using (2.10) and the Fubini theorem,

$$
\begin{array}{r}
A_{1}(\zeta)=E\left[\int _ { 0 } ^ { T } Z ( t ^ { - } ) \left(\int_{0}^{t^{-}} Z^{-1}\left(s^{-}\right) \lambda(s) d \zeta(s)\right.\right. \\
\left.\left.\quad+\sum_{0<s<t} Z^{-1}\left(s^{-}\right) \lambda(s) \alpha(s) \Delta \zeta(s)\right) d Q(t)\right] \\
=E\left[\int_{0}^{T}\left(\int_{t^{+}}^{T} Z\left(s^{-}\right) d Q(s)\right) Z^{-1}(t) \lambda(t) d \zeta(t)\right. \\
\left.\quad+\sum_{0<t \leq T}\left(\int_{t^{+}}^{T} Z\left(s^{-}\right) d Q(s)\right) Z^{-1}(t) \lambda(t) \alpha(t) \Delta \zeta(t)\right],
\end{array}
$$

where

$$
d Q(s)=\frac{\partial H_{0}}{\partial x}(s) d s+R(s) \frac{\partial \lambda}{\partial x}(s) d \xi(s)
$$

We thus get, using (2.14),

$$
\begin{aligned}
\lim _{y \rightarrow 0^{+}} \frac{1}{y}(J(\xi+y \zeta)-J(\xi)) \\
=E\left[\int_{0}^{T}[\lambda(t) \tilde{p}(t)+h(t)] d \zeta(t)+\sum_{0<t \leq T} \lambda(t) S(t) \alpha(t) \Delta \zeta(t)\right] \\
=E\left[\int_{0}^{T}[\lambda(t) \tilde{p}(t)+h(t)] d \zeta^{c}(t)\right. \\
\left.\quad+\sum_{0<t \leq T}\{\lambda(t)(\tilde{p}(t)+S(t) \alpha(t))+h(t)\} \Delta \zeta(t)\right] .
\end{aligned}
$$

This completes the proof of Lemma 2.3. $\quad \square$

We can now prove the main result of this section.

Theorem 2.4 (maximum principle I). Set

$$
\begin{aligned}
U(t) & =U_{\xi}(t)=\lambda(t) \tilde{p}(t)+h(t), \\
V(t) & =V_{\xi}(t)=\lambda(t)(\tilde{p}(t)+S(t) \alpha(t))+h(t) ; t \in[0, T] .
\end{aligned}
$$

(i) Suppose $\xi \in \mathcal{A}_{\mathcal{E}}$ is optimal for problem (2.4). Then a.a. $t \in[0, T]$, we have

$$
E\left[U(t) \mid \mathcal{E}_{t}\right] \leq 0 \quad \text { and } E\left[U(t) \mid \mathcal{E}_{t}\right] d \xi^{c}(t)=0
$$

and for all $t \in[0, T]$ we have

$$
E\left[V(t) \mid \mathcal{E}_{t}\right] \leq 0 \quad \text { and } E\left[V(t) \mid \mathcal{E}_{t}\right] \Delta \xi(t)=0 .
$$

Copyright $@$ by SIAM. Unauthorized reproduction of this article is prohibited. 
(ii) Conversely, suppose (2.30) and (2.31) hold for some $\xi \in \mathcal{A}_{\mathcal{E}}$. Then $\xi$ is a directional substationary point for $J(\xi)$ in the sense that

$$
\lim _{y \rightarrow 0^{+}} \frac{1}{y}(J(\xi+y \zeta)-J(\xi)) \leq 0 \quad \text { for all } \zeta \in \mathcal{V}(\xi)
$$

Proof. (i) Suppose $\xi$ is optimal for problem (2.4). Then

$$
\lim _{y \rightarrow 0^{+}} \frac{1}{y}(J(\xi+y \zeta)-J(\xi)) \leq 0 \quad \text { for all } \zeta \in \mathcal{V}(\xi) .
$$

Hence, by Lemma 2.3,

$$
E\left[\int_{0}^{T} U(t) d \zeta^{c}(t)+\sum_{0<t \leq T} V(t) \Delta \zeta(t)\right] \leq 0 \quad \text { for all } \zeta \in \mathcal{V}(\xi)
$$

In particular, this holds if we fix $t \in[0, T]$ and choose $\zeta$ such that

$$
d \zeta(s)=a(\omega) \delta_{t}(s) ; s \in[0, T]
$$

where $a(\omega) \geq 0$ is $\mathcal{E}_{t}$-measurable and bounded and $\delta_{t}($.$) is the unit point mass at t.$ Then (2.33) gets the form

$$
E[V(t) a] \leq 0
$$

Since this holds for all bounded $\mathcal{E}_{t}$-measurable $a \geq 0$, we conclude that

$$
E\left[V(t) \mid \mathcal{E}_{t}\right] \leq 0
$$

Next, choose $\zeta(t)=-\xi^{d}(t)$, the purely discontinuous part of $\xi$. Then clearly $\zeta \in \mathcal{V}(\xi)$ (with $\delta=1$ ), so by $(2.33)$ we get

$$
E\left[\sum_{0<t \leq T} V(t)(-\Delta \xi(t))\right] \leq 0
$$

On the other hand, choosing $\zeta=\xi^{d}$ in (2.33) gives

$$
E\left[\sum_{0<t \leq T} V(t) \Delta \xi(t)\right] \leq 0 .
$$

Combining (2.35) and (2.36) we obtain

$$
E\left[\sum_{0<t \leq T} E\left[V(t) \mid \mathcal{E}_{t}\right] \Delta \xi(t)\right]=E\left[\sum_{0<t \leq T} V(t) \Delta \xi(t)\right]=0 .
$$

Copyright (c) by SIAM. Unauthorized reproduction of this article is prohibited. 
Since $E\left[V(t) \mid \mathcal{E}_{t}\right] \leq 0$ and $\Delta \xi(t) \geq 0$, this implies that

$$
E\left[V(t) \mid \mathcal{E}_{t}\right] \Delta \xi(t)=0
$$

for all $t \in[0, T]$, as claimed. This proves (2.31).

To prove (2.30) we proceed similarly. First choosing

$$
d \zeta(t)=a(t) d t ; \quad t \in[0, T],
$$

where $a(t) \geq 0$ is continuous and $\mathcal{E}_{t}$-adapted we get from (2.33) that

$$
E\left[\int_{0}^{T} U(t) a(t) d t\right] \leq 0
$$

Since this holds for all such $\mathcal{E}_{t}$-adapted processes we deduce that

$$
E\left[U(t) \mid \mathcal{E}_{t}\right] \leq 0 ; \text { a.a. } t \in[0, T] .
$$

Then, choosing $\zeta(t)=-\xi^{c}(t)$ we get from (2.33) that

$$
E\left[\int_{0}^{T} U(t)\left(-d \xi^{c}(t)\right)\right] \leq 0 .
$$

Next, choosing $\zeta(t)=\xi^{c}(t)$ we get

$$
E\left[\int_{0}^{T} U(t) d \xi^{c}(t)\right] \leq 0
$$

Hence

$$
E\left[\int_{0}^{T} U(t) d \xi^{c}(t)\right]=E\left[\int_{0}^{T} E\left[U(t) \mid \mathcal{E}_{t}\right] d \xi^{c}(t)\right]=0,
$$

which combined with (2.38) gives

$$
E\left[U(t) \mid \mathcal{E}_{t}\right] d \xi^{c}(t)=0
$$

(ii) Suppose (2.30) and (2.31) hold for some $\xi \in \mathcal{A}_{\mathcal{E}}$. Choose $\zeta \in \mathcal{V}(\xi)$. Then $\xi+y \zeta \in \mathcal{A}_{\mathcal{E}}$ and hence $d \xi+y d \zeta \geq 0$ for all $y \in[0, \delta]$ for some $\delta>0$. Therefore,

$$
\begin{aligned}
y E & {\left[\int_{0}^{T} U(t) d \zeta^{c}(t)+\sum_{0<t \leq T} V(t) \Delta \zeta(t)\right] } \\
= & y E\left[\int_{0}^{T} E\left[U(t) \mid \mathcal{E}_{t}\right] d \zeta^{c}(t)+\sum_{0<t \leq T} E\left[V(t) \mid \mathcal{E}_{t}\right] \Delta \zeta(t)\right] \\
= & E\left[\int_{0}^{T} E\left[U(t) \mid \mathcal{E}_{t}\right] d \xi^{c}(t)+\sum_{0<t \leq T} E\left[V(t) \mid \mathcal{E}_{t}\right] \Delta \xi(t)\right] \\
& +y E\left[\int_{0}^{T} E\left[U(t) \mid \mathcal{E}_{t}\right] d \zeta^{c}(t)+\sum_{0<t \leq T} E\left[V(t) \mid \mathcal{E}_{t}\right] \Delta \zeta(t)\right] \\
= & E\left[\int_{0}^{T} E\left[U(t) \mid \mathcal{E}_{t}\right] d\left(\xi^{c}(t)+y \zeta^{c}(t)\right)+\sum_{0<t \leq T} E\left[V(t) \mid \mathcal{E}_{t}\right] \Delta(\xi+y \zeta)(t)\right] \leq 0
\end{aligned}
$$

by (2.30)-(2.31). Hence the conclusion follows from Lemma 2.3 .

Copyright (c) by SIAM. Unauthorized reproduction of this article is prohibited. 
Remark 2.5. Note that if $\frac{\partial \theta}{\partial x}(s, z)=\frac{\partial \lambda}{\partial x}(s, x)=0$ for all $s, z, x$, then $\alpha(s)=0$ and hence $U(s)=V(s)$. Therefore, in this case, conditions (2.30)-(2.31) reduce to the condition

$$
E\left[U(t) \mid \mathcal{E}_{t}\right] \leq 0 \text { and } E\left[U(t) \mid \mathcal{E}_{t}\right] d \xi(t)=0
$$

Markovian case. Equation (2.30) is a pathwise version of the variational inequalities in the (monotone) singular control problem in the classical Markovian and full information $\left(\mathcal{E}_{t}=\mathcal{F}_{t}\right)$ jump diffusion setting. Indeed we have in this case (in dimension 1)

$$
d X(t)=b(t, X(t)) d t+\sigma(t, X(t)) d B(t)+\int_{\mathbb{R}_{0}} \theta\left(t, X\left(t^{-}\right), z\right) \tilde{N}(d t, d z)+\lambda(t) d \xi(t)
$$

and

$$
J^{\xi}(t, x)=E^{t, x}\left[\int_{t}^{T} f(s, X(s)) d s+g(X(T))+\int_{t}^{T} h\left(s, X\left(s^{-}\right)\right) d \xi(s)\right],
$$

where $b: \mathbb{R}^{2} \rightarrow \mathbb{R}, \sigma: \mathbb{R}^{2} \rightarrow \mathbb{R}, \theta: \mathbb{R}^{2} \times \mathbb{R}_{0} \rightarrow, \lambda: \mathbb{R}^{2} \rightarrow \mathbb{R}, f: \mathbb{R}^{2} \rightarrow \mathbb{R}, g: \mathbb{R} \rightarrow \mathbb{R}$, and $h: \mathbb{R}^{2} \rightarrow \mathbb{R}$ are given deterministic functions. Define

$$
\begin{aligned}
A \varphi(t, x)= & \frac{\partial \varphi}{\partial t}+b(t, x) \frac{\partial \varphi}{\partial x}+\frac{1}{2} \sigma^{2}(t, x) \frac{\partial^{2} \varphi}{\partial x^{2}} \\
& +\int_{\mathbb{R}_{0}}\left\{\varphi(t, x+\theta(t, x, z))-\varphi(t, x)-\theta(t, x, z) \frac{\partial \varphi}{\partial x}(t, x)\right\} \nu(d z) .
\end{aligned}
$$

Then the variational inequalities for the value function $\varphi(t, x)=\sup _{\xi \in \mathcal{A}_{\mathcal{E}}} J^{\xi}(t, x)$ are (see, e.g., [16, Theorem 6.2])

$$
\begin{aligned}
A \varphi(t, x)+f(t, x) & \leq 0 \text { for all } t, x, \\
\lambda(t) \frac{\partial \varphi}{\partial x}(t, x)+h(t, x) & \leq 0 \text { for all } t, x
\end{aligned}
$$

with the boundary condition $\varphi(T, x)=g(x)$.

Let $D=\left\{(t, x) ; \lambda(t) \frac{\partial \varphi}{\partial x}(t, x)+h(t, x)<0\right\}$ be the continuation region. Then

$$
\begin{aligned}
A \varphi(t, x)+f(t, x) & =0 \text { in } D \\
(t, \hat{X}(t)) & \in \bar{D} \text { for all } t \\
\left\{\lambda(t) \frac{\partial \varphi}{\partial x}(t, \hat{X}(t))+h(t, \hat{X}(t))\right\} d \hat{\xi}^{c}(t) & =0 \text { for all } t, \text { a.s. } \\
\left\{\Delta_{\hat{\xi}} \varphi(t, \hat{X}(t))+h(t, \hat{X}(t))\right\} \Delta \hat{\xi}(t) & =0 \text { for all } t, \text { a.s }
\end{aligned}
$$

Copyright (c) by SIAM. Unauthorized reproduction of this article is prohibited. 
where $\hat{X}(t)=X^{\hat{\xi}}(t)$ is the process corresponding to the optimal control $\hat{\xi}$ and $\Delta_{\hat{\xi}} \varphi(t, \hat{X}(t))$ is the jump of $\varphi(t, \hat{X}(t))$ due to the jump in $\hat{\xi}$ at time $t$.

Hence, comparing with Theorem 2.4 we see that $\lambda(t) \frac{\partial \varphi}{\partial x}(t, X(t))+h(t, X(t))$ corresponds to $\lambda(t) E\left[\tilde{p}(t) \mid \mathcal{F}_{t}\right]+h(t, X(t))$ which means that $\frac{\partial \varphi}{\partial x}(t, X(t))$ corresponds to $E\left[\tilde{p}(t) \mid \mathcal{F}_{t}\right]$.

2.3. A Hamiltonian-based maximum principle. We now present an alternative way of computing the right-sided derivative of (2.6) for the computation of

$$
\lim _{y \rightarrow 0^{+}} \frac{1}{y}(J(\xi+y \zeta)-J(\xi)) \quad \text { for } \xi \in \mathcal{A}_{\mathcal{E}}, \zeta \in \mathcal{V}(\xi)
$$

The method is based on using a singular control version of the Hamiltonian as follows.

Define the stochastic differential Hamiltonian

$$
H(t, x, p, q, r(.))(d t, d \xi):[0, T] \times \mathbb{R} \times \mathbb{R} \times \mathbb{R} \times \mathcal{R} \mapsto \mathcal{M}
$$

by

$$
\begin{aligned}
& H(t, x, p, q, r(.))(d t, d \xi) \\
&=\left\{f(t, x)+p b(t, x)+q \sigma(t, x)+\int_{\mathbb{R}_{0}} r(t, z) \theta(t, x, z) \nu(d z)\right\} d t \\
&+\{p \lambda(t, x)+h(t, x)\} d \xi(t)+\lambda(t, x) \int_{\mathbb{R}_{0}} r(t, z) N(\{t\}, d z) \Delta \xi(t) .
\end{aligned}
$$

Here $\mathcal{R}$ is the set of functions $r():. \mathbb{R}_{0} \mapsto \mathbb{R}$ such that (2.49) is well defined and $\mathcal{M}$ is the set of all sums of stochastic $d t-$ and $d \xi(t)$ - differentials, $\xi \in \mathcal{A}_{\mathcal{E}}$.

Let $\xi \in \mathcal{A}_{\mathcal{E}}$ with associated process $X(t)=X^{\xi}(t)$. The triple of $\mathcal{F}_{t}$-adapted adjoint processes $(p(t), q(t), r(t, z))=\left(p_{\xi}(t), q_{\xi}(t), r_{\xi}(t, z)\right)$ associated to $\xi$ are given by the following BSDE:

$$
\begin{aligned}
d p(t)= & -\frac{\partial H}{\partial x}\left(t, X\left(t^{-}\right), p\left(t^{-}\right), q\left(t^{-}\right), r\left(t^{-}, \cdot\right)\right)(d t, d \xi(t)) \\
& +q(t) d B(t)+\int_{\mathbb{R}_{0}} r(t, z) \tilde{N}(d t, d z) ; 0 \leq t<T \\
p(T)= & g^{\prime}(X(T)) .
\end{aligned}
$$

Solving this equation provides a relation between the adjoint process $p$ and $\tilde{p}$ given by $(2.17)$.

Proposition 2.6. Let $\tilde{p}(t)$ be the process given by $(2.17)$ and let $p(t)$ be the adjoint process given by the BSDE (2.50). Then

$$
p(t)=E\left[\tilde{p}(t) \mid \mathcal{F}_{t}\right]
$$

Copyright $@$ by SIAM. Unauthorized reproduction of this article is prohibited. 
Proof. The BSDE (2.50) for $p(t)$ is linear and its solution is

$$
p(t)=E\left[g^{\prime}(X(T)) G(t, T)+\int_{t^{+}}^{T} G\left(t, s^{-}\right)\left\{\frac{\partial f}{\partial x}(s) d s+\frac{\partial h}{\partial x}\left(s^{-}\right) d \xi(s)\right\} \mid \mathcal{F}_{t}\right]
$$

where $G(t, s)$ is defined in (2.14). Hence, by (2.12),

$$
\begin{aligned}
Z(t) p(t)= & E\left[g^{\prime}(X(T)) Z(T)+\int_{t^{+}}^{T} Z(s)\left\{\frac{\partial f}{\partial x}(s) d s+\frac{\partial h}{\partial x}(s) d \xi(s)\right\} \mid \mathcal{F}_{t}\right] \\
= & E\left[g ^ { \prime } ( X ( T ) ) \left(Z(t)+\int_{t}^{T} Z\left(u^{-}\right)\left\{\frac{\partial b}{\partial x}(u) d u+\frac{\partial \sigma}{\partial x}(u) d B(u)\right.\right.\right. \\
& \left.\left.+\int_{\mathbb{R}_{0}} \frac{\partial \theta}{\partial x}(u, z) \tilde{N}(d u, d z)+\frac{\partial \lambda}{\partial x}(u) d \xi(u)\right\}\right) \\
& +\int_{t^{+}}^{T}\left(Z(t)+\int_{t}^{s} Z\left(u^{-}\right)\left\{\frac{\partial b}{\partial x}(u) d u+\frac{\partial \sigma}{\partial x}(u) d B(u)\right.\right. \\
& \left.\left.\left.\left(\frac{\partial f}{\partial x}(s) d s+\frac{\partial h}{\partial x}(s) d \xi(s)\right) \mid \mathcal{F}_{t}\right] \frac{\partial \theta}{\partial x}(u, z) \tilde{N}(d u, d z)+\frac{\partial \lambda}{\partial x}(u) d \xi(u)\right\}\right) \\
= & E\left[Z(t) R(t)+g^{\prime}(X(T)) \int_{t}^{T} Z\left(s^{-}\right)\left\{\frac{\partial b}{\partial x}(s) d s+\frac{\partial \sigma}{\partial x}(s) d B(s)\right.\right. \\
& +\int_{t}^{T}\left(\int_{u}^{T} \frac{\partial f}{\partial x}(s) d s+\frac{\partial h}{\partial x}(s) d \xi(s)\right) \\
& +u_{\mathbb{R}_{0}^{-}} \frac{\partial b}{\partial x}(u) d u+\frac{\partial \sigma}{\partial x}(u) d B(u) \\
& \left.+\int_{\mathbb{R}_{0}} \frac{\partial \theta}{\partial x}(s, z) \tilde{N}(d s, d z)+\frac{\partial \lambda}{\partial x}(s) d \xi(s)\right\}
\end{aligned}
$$

Copyright $\odot$ by SIAM. Unauthorized reproduction of this article is prohibited. 
By the duality formulae this is equal to

$$
\begin{gathered}
E\left[Z(t) R(t)+\int_{t}^{T}\left(Z(s) R(s) \frac{\partial b}{\partial x}(s) d s+Z(s) R(s) \frac{\partial \lambda}{\partial x}(s) d \xi(s)+D_{s^{+}}(Z(s) R(s)) \frac{\partial \sigma}{\partial x}(s) d s\right.\right. \\
\left.\left.+\int_{\mathbb{R}_{0}} D_{s^{+}, z}(Z(s) R(s)) \frac{\partial \theta}{\partial x}(s, z) \nu(d z) d s\right) \mid \mathcal{F}_{t}\right] \\
=Z(t) E\left[R(t)+\int_{t}^{T} G(t, s)\left(R(s) \frac{\partial b}{\partial x}(s) d s+R(s) \frac{\partial \lambda}{\partial x}(s) d \xi(s)+D_{s^{+}} R(s) \frac{\partial \sigma}{\partial x}(s) d s\right.\right. \\
\left.\left.\quad+\int_{\mathbb{R}_{0}} D_{s^{+}, z} R(s) \frac{\partial \theta}{\partial x}(s, z) \nu(d z) d s\right) \mid \mathcal{F}_{t}\right] \\
=Z(t) E\left[\tilde{p}(t) \mid \mathcal{F}_{t}\right] \text { by }(2.17) . \quad
\end{gathered}
$$

In the following as well as in section 2.4, we assume

$$
\frac{\partial \lambda}{\partial x}(t, x)=\frac{\partial h}{\partial x}(t, x)=0 \text { for all } t, x
$$

The following result is analogous to Lemma 2.3.

Lemma 2.7. Assume (2.53) holds. Let $\xi \in \mathcal{A}_{\mathcal{E}}$ and $\zeta \in \mathcal{V}(\xi)$. Put

$$
\eta=\xi+y \zeta \quad \text { for } y \in[0, \delta(\xi)]
$$

Assume that

$$
\begin{aligned}
& E\left[\int _ { 0 } ^ { T } \left\{\left|X^{\eta}(t)-X^{\xi}(t)\right|^{2}\left(q_{\xi}^{2}(t)+\int_{\mathbb{R}_{0}} r_{\xi}^{2}(t, z) \nu(d z)\right)+p_{\xi}^{2}(t)\left(\mid \sigma\left(t, X^{\eta}(t)-\left.\sigma\left(t, X^{\xi}(t)\right)\right|^{2}\right.\right.\right.\right. \\
& \left.\left.(2.54)+\int_{\mathbb{R}_{0}}\left|\theta\left(t, X^{\eta}(t), z\right)-\theta\left(t, X^{\xi}(t), z\right)\right|^{2} \nu(d z)\right\} d t\right]<\infty \text { for all } y \in[0, \delta(\xi)] .
\end{aligned}
$$

Then

$$
\begin{aligned}
& \lim _{y \rightarrow 0^{+}} \frac{1}{y}(J(\xi+y \zeta)-J(\xi)) \\
&55)=E\left[\int_{0}^{T}(\lambda(t) p(t)+h(t)) d \zeta(t)+\sum_{0<t \leq T} \lambda(t) \int_{\mathbb{R}_{0}} r(t, z) N(\{t\}, d z) \Delta \zeta(t)\right] .
\end{aligned}
$$

Proof. We compute the r.h.s. of (2.6). By the definition of $H$, we have

$$
\begin{aligned}
E\left[\int_{0}^{T} \frac{\partial f}{\partial x}(t) \mathcal{Y}(t) d t\right]=E\left[\int_{0}^{T} \mathcal{Y}\left(t^{-}\right)\right. & \left(\frac{\partial H}{\partial x}(d t, d \xi)-p(t) \frac{\partial b}{\partial x}(t) d t-q(t) \frac{\partial \sigma}{\partial x}(t) d t\right. \\
& \left.\left.-\int_{\mathbb{R}_{0}} r(t, z) \frac{\partial \theta}{\partial x}(t, z) \nu(d z) d t\right)\right]
\end{aligned}
$$

Copyright $@$ by SIAM. Unauthorized reproduction of this article is prohibited. 
By the equations for $p(t)$ and $\mathcal{Y}(t)$,

$$
\begin{aligned}
& E\left[g^{\prime}(X(T)) \mathcal{Y}(T)\right] \\
& =E[p(T) \mathcal{Y}(T)]=E\left[\int_{0}^{T} \mathcal{Y}\left(t^{-}\right) d p(t)+\int_{0}^{T} p\left(t^{-}\right) d \mathcal{Y}(t)\right. \\
& +\int_{0}^{T} \mathcal{Y}(t) \frac{\partial \sigma}{\partial x}(t) q(t) d t+\int_{0}^{T} \int_{\mathbb{R}_{0}} \mathcal{Y}(t) \frac{\partial \theta}{\partial x}(t, z) r(t, z) \nu(d z) d t \\
& \left.+\sum_{0<t \leq T} \lambda(t) \int_{\mathbb{R}_{0}} r(t, z) N(\{t\}, d z) \Delta \zeta(t)\right] \\
& =E\left[\int_{0}^{T} \mathcal{Y}\left(t^{-}\right)\left\{-\frac{\partial H}{\partial x}(d t, d \xi)\right\}+\int_{0}^{T} p\left(t^{-}\right) \mathcal{Y}(t) \frac{\partial b}{\partial x}(t) d t+\int_{0}^{T} p(t) \lambda(t) d \zeta(t)\right. \\
& +\int_{0}^{T} \mathcal{Y}(t) \frac{\partial \sigma}{\partial x}(t) q(t) d t+\int_{0}^{T} \int_{\mathbb{R}_{0}} \mathcal{Y}(t) \frac{\partial \theta}{\partial x}(t, z) r(t, z) \nu(d z) d t \\
& \left.(2.57)+\sum_{0<t \leq T} \lambda(t) \int_{\mathbb{R}_{0}} r(t, z) N(\{t\}, d z) \Delta \zeta(t)\right] .
\end{aligned}
$$

Summing up (2.56)-(2.57) and using (2.6) we get (2.55), as claimed.

Proceeding as in the proof of Theorem 2.4, we obtain the following.

Theorem 2.8 (maximum principle II). (i) Suppose $\xi \in \mathcal{A}_{\mathcal{E}}$ is optimal for problem (2.4) and that (2.53) and (2.54) hold. Then

$$
E\left[p(t) \lambda(t)+h(t) \mid \mathcal{E}_{t}\right] \leq 0 ; \quad E\left[p(t) \lambda(t)+h(t) \mid \mathcal{E}_{t}\right] d \xi^{c}(t)=0 \text { for all } t
$$

and

$$
\begin{array}{r}
E\left[\lambda(t)\left(p(t)+\int_{\mathbb{R}_{0}} r(t, z) N(\{t\}, d z)\right)+h(t) \mid \mathcal{E}_{t}\right] \leq 0 ; \\
E\left[\lambda(t)\left(p(t)+\int_{\mathbb{R}_{0}} r(t, z) N(\{t\}, d z)\right)+h(t) \mid \mathcal{E}_{t}\right] \Delta \xi(t)=0 .
\end{array}
$$

(ii) Conversely, suppose (2.54) and (2.58)-(2.60) hold. Then $\xi$ is a directional substationary point for $J(\xi)$ in the sense that $\lim _{y \rightarrow 0^{+}} \frac{1}{y}(J(\xi+y \zeta)-J(\xi)) \leq 0$ for all $\zeta \in \mathcal{V}(\xi)$.

2.4. A Mangasarian (sufficient) maximum principle. The results of the previous sections have been of the type of "necessary" conditions for a control to be optimal in the sense that they state that if a given control is optimal, then a certain Hamiltonian functional is maximized. In this section we give sufficient conditions for optimality. We do this in terms of the stochastic differential Hamiltonian $H$ and the adjoint processes $p(t), q(t), r(t, z)$ defined in (2.49) and (2.50), in the case when $\lambda$ and $h$ do not depend on $x$.

Theorem 2.9 (Mangasarian maximum principle). Assume that

- (2.53) holds,

- $x \rightarrow g(x)$ is concave,

- there exists a feedback control $\hat{\xi}=\hat{\xi}(x, d t) \in \mathcal{A}_{\mathcal{E}}$ with corresponding solution $\hat{X}(t)=X^{\hat{\xi}}(t)$ of (2.1) and $\hat{p}(t), \hat{q}(t), \hat{r}(t, z)$ of $(2.50)$ such that

$$
\hat{\xi}(x) \in \operatorname{argmax}_{\xi \in \mathcal{A}_{\mathcal{E}}} E\left[H\left(t, x, \hat{p}\left(t^{-}\right), \hat{q}\left(t^{-}\right), \hat{r^{-}}(t, \cdot)\right)(d t, d \xi(t)) \mid \mathcal{E}_{t}\right],
$$

Copyright $@$ by SIAM. Unauthorized reproduction of this article is prohibited. 
i.e.,

$$
\begin{aligned}
& E\left[\hat{p}(t) \lambda(t)+h(t) \mid \mathcal{E}_{t}\right] d \xi(t)+\lambda(t) E\left[\int_{\mathbb{R}_{0}} \hat{r}(t, z) N(\{t\}, d z) \mid \mathcal{E}_{t}\right] \Delta \xi(t) \\
& \leq E\left[\hat{p}(t) \lambda(t)+h(t) \mid \mathcal{E}_{t}\right] d \hat{\xi}(t)+\lambda(t) E\left[\int_{\mathbb{R}_{0}} \hat{r}(t, z) N(\{t\}, d z) \mid \mathcal{E}_{t}\right] \Delta \hat{\xi}(t) \\
& \quad \text { for all } \xi \in \mathcal{A}_{\mathcal{E}},
\end{aligned}
$$

- $\hat{h}(x):=E\left[H\left(t, x, \hat{p}\left(t^{-}\right), \hat{q}\left(t^{-}\right), \hat{r}\left(t^{-}, \cdot\right)\right)(d t, d \hat{\xi}(t)) \mid \mathcal{E}_{t}\right]$ is a concave function of $x$ (the Arrow condition),

$$
\begin{aligned}
& E\left[\int _ { 0 } ^ { T } \left\{|X(t)-\hat{X}(t)|^{2}\left(\hat{q}^{2}(t)+\int_{\mathbb{R}_{0}} \hat{r}^{2}(t, z) \nu(d z)\right)\right.\right. \\
& \quad+\hat{p}(t)^{2}\left(|\sigma(t, X(t))-\sigma(t, \hat{X}(t))|^{2}\right. \\
& \left.\left.(2.61)+\int_{\mathbb{R}_{0}}|\theta(t, X(t), z)-\theta(t, \hat{X}(t), z)|^{2} \nu(d z)\right\} d t\right]<\infty \quad \text { for all } \xi \in \mathcal{A}_{\mathcal{E}} .
\end{aligned}
$$

Then $\hat{\xi}$ is an optimal control for problem (2.4).

Proof. Choose $\xi \in \mathcal{A}_{\mathcal{E}}$ and consider with $X=X^{\xi}$

$$
J(\xi)-J(\hat{\xi})=I_{1}+I_{2}+I_{3}
$$

where

$$
\begin{aligned}
& I_{1}=E\left[\int_{0}^{T}\{f(t, X(t))-f(t, \hat{X}(t))\} d t\right] \\
& I_{2}=E[g(X(T))-g(\hat{X}(T))] \\
& I_{3}=E\left[\int_{0}^{T}\{h(t) d \xi(t)-h(t) d \hat{\xi}(t)\}\right]
\end{aligned}
$$

By our definition of $H$ we have

$$
\begin{aligned}
I_{1}=E[ & \int_{0}^{T}\left\{H\left(t, X\left(t^{-}\right), \hat{p}\left(t^{-}\right), \hat{q}\left(t^{-}\right), \hat{r^{-}}(t, \cdot)\right)(d t, d \xi)\right. \\
& \left.-H\left(t, \hat{X}\left(t^{-}\right), \hat{p}\left(t^{-}\right), \hat{q}\left(t^{-}\right), \hat{r}\left(t^{-}, \cdot\right)\right)(d t, d \hat{\xi})\right\} \\
& -\int_{0}^{T}\{b(t, X(t))-b(t, \hat{X}(t))\} \hat{p}(t) d t-\int_{0}^{T}\{\sigma(t, X(t))-\sigma(t, \hat{X}(t))\} \hat{q}(t) d t \\
& -\int_{0}^{T} \int_{\mathbb{R}_{0}}\{\theta(t, X(t), z)-\theta(t, \hat{X}(t), z)\} \hat{r}(t, z) \nu(d z) d t \\
& -\int_{0}^{T} \hat{p}\left(t^{-}\right)\{\lambda(t) d \xi(t)-\lambda(t) d \hat{\xi}(t)\}-\int_{0}^{T}\{h(t) d \xi(t)-h(t) d \hat{\xi}(t)\} \\
& \left.-\sum_{0<t \leq T} \lambda(t) \int_{\mathbb{R}_{0}} \hat{r}(t, z) N(\{t\}, d z)(\Delta \xi(t)-\Delta \hat{\xi}(t))\right] .
\end{aligned}
$$

Copyright $@$ ( ) by SIAM. Unauthorized reproduction of this article is prohibited. 
By concavity of $g$ and (2.50)

$$
\begin{aligned}
& I_{2} \leq E[\left.g^{\prime}(\hat{X}(T))(X(T)-\hat{X}(T))\right]=E[\hat{p}(T)(X(T)-\hat{X}(T))] \\
&=E\left[\int_{0}^{T}\left\{X\left(t^{-}\right)-\hat{X}\left(t^{-}\right)\right\} d \hat{p}(t)+\int_{0}^{T} \hat{p}\left(t^{-}\right)(d X(t)-d \hat{X}(t))\right. \\
& \quad+\int_{0}^{T}\{\sigma(t, X(t))-\sigma(t, \hat{X}(t))\} \hat{q}(t) d t \\
& \quad+\int_{0}^{T} \int_{\mathbb{R}_{0}}\{\theta(t, X(t), z)-\theta(t, \hat{X}(t), z)\} \hat{r}(t, z) \nu(d z) d t \\
&\left.\quad+\sum_{0<t \leq T} \lambda(t) \int_{\mathbb{R}_{0}} \hat{r}(t, z) N(\{t\}, d z)(\Delta \xi(t)-\Delta \hat{\xi}(t))\right] \\
&=E\left[\int_{0}^{T}\left(X\left(t^{-}\right)-\hat{X}\left(t^{-}\right)\right)\left\{-\frac{\partial H}{\partial x}\left(t, \hat{X}\left(t^{-}\right), \hat{p}\left(t^{-}\right), \hat{q}\left(t^{-}\right), \hat{r}\left(t^{-}, \cdot\right)\right)(d t, d \xi(t))\right\}\right. \\
& \quad+\int_{0}^{T} \hat{p}\left(t^{-}\right)\{b(t, X(t))-b(t, \hat{X}(t))\} d t+\int_{0}^{T} \hat{p}\left(t^{-}\right)\{\lambda(t) d \xi(t)-\lambda(t) d \hat{\xi}(t)\} \\
& \quad+\int_{0}^{T}\{\sigma(t, X(t))-\sigma(t, \hat{X}(t))\} \hat{q}(t) d t \\
& \quad+\int_{0}^{T} \int_{\mathbb{R}_{0}}\{\theta(t, X(t), z)-\theta(t, \hat{X}(t), z)\} \hat{r}(t, z) \nu(d z) d t \\
&\left.\quad+\quad \sum_{0<t \leq T} \lambda(t) \int_{\mathbb{R}_{0}} \hat{r}(t, z) N(\{t\}, d z)(\Delta \xi(t)-\Delta \hat{\xi}(t))\right] .
\end{aligned}
$$

Combining (2.62)-(2.69) we get, using concavity of $H$,

$$
\begin{aligned}
& J(\xi)-J(\hat{\xi}) \leq E {\left[\int _ { 0 } ^ { T } \left\{H\left(t, X\left(t^{-}\right), \hat{p}\left(t^{-}\right), \hat{q}\left(t^{-}\right), \hat{r}\left(t^{-}, \cdot\right)\right)(d t, d \xi(t))\right.\right.} \\
&\left.-H\left(t, \hat{X}\left(t^{-}\right), \hat{p}\left(t^{-}\right), \hat{q}\left(t^{-}\right), \hat{r}\left(t^{-}, \cdot\right)\right)(t, \cdot)\right)(d t, d \hat{\xi}(t)) \\
&\left.\left.(2.70) \quad-\left(X\left(t^{-}\right)-\hat{X}\left(t^{-}\right)\right) \frac{\partial H}{\partial x}\left(t, \hat{X}\left(t^{-}\right), \hat{p}\left(t^{-}\right), \hat{q}\left(t^{-}\right), \hat{r}\left(t^{-}, \cdot\right)\right)(d t, d \hat{\xi}(t))\right\}\right] .
\end{aligned}
$$

Since $\hat{h}(x)$ is concave, it follows by a standard separating hyperplane argument (see, e.g., $[20$, Chapter 5 , section 23]) that there exists a supergradient $a \in \mathbb{R}$ for $\hat{h}(x)$ at $x=\hat{X}\left(t^{-}\right)$, i.e.,

$$
\hat{h}(x)-\hat{h}\left(\hat{X}\left(t^{-}\right)\right) \leq a\left(x-\hat{X}\left(t^{-}\right)\right) \text {for all } x .
$$

Define

$$
\varphi(x)=\hat{h}(x)-\hat{h}\left(\hat{X}\left(t^{-}\right)\right)-a\left(x-\hat{X}\left(t^{-}\right)\right) \quad x \in \mathbb{R} .
$$

Then

$$
\varphi(x) \leq 0 \text { for all } x
$$

and

$$
\varphi\left(\hat{X}\left(t^{-}\right)\right)=0
$$

Copyright (c) by SIAM. Unauthorized reproduction of this article is prohibited. 
Hence

$$
\varphi^{\prime}\left(\hat{X}\left(t^{-}\right)\right)=0
$$

which implies that

$$
\frac{\partial H}{\partial x}\left(t, \hat{X}\left(t^{-}\right), \hat{p}\left(t^{-}\right), \hat{q}\left(t^{-}\right), \hat{r}\left(t^{-}, \cdot\right)\right)(d t, d \hat{\xi}(t))=\frac{\partial \hat{h}}{\partial x}\left(\hat{X}\left(t^{-}\right)\right)=a .
$$

Combining this with (2.70) we get

$$
\begin{aligned}
J(\xi)-J(\hat{\xi}) & \leq \hat{h}\left(X\left(t^{-}\right)\right)-\hat{h}\left(\hat{X}\left(t^{-}\right)\right)-\left(X\left(t^{-}\right)-\hat{X}\left(t^{-}\right)\right) \frac{\partial \hat{h}}{\partial x}\left(\hat{X}\left(t^{-}\right)\right) \\
& \leq 0, \quad \text { since } \hat{h}(x) \text { is concave. }
\end{aligned}
$$

This proves that $\hat{\xi}$ is optimal.

2.5. A special case. From now on, we restrict ourselves to the case when

$$
\frac{\partial b}{\partial x}=\frac{\partial \sigma}{\partial x}=\frac{\partial \theta}{\partial x}=\frac{\partial \lambda}{\partial x}=0 \quad \text { and } \quad \lambda(t, x) \equiv \lambda(t)<0 \text { a.s. for all } t \in[0, T] .
$$

We thus consider a controlled singular Itô-Lévy process $X^{\xi}(t)$ of the form $X^{\xi}(0)=x$ and

$$
d X^{\xi}(t)=b(t) d t+\sigma(t) d B(t)+\int_{\mathbb{R}_{0}} \theta(t, z) \tilde{N}(d t, d z)+\lambda(t) d \xi(t) ; t \in[0, T],
$$

where $b(t), \sigma(t), \theta(t, z)$ are given $\mathcal{F}_{t}$-predictable processes for all $z \in \mathbb{R}_{0}$. We denote by $X^{0}(t)$ the uncontrolled state process, that is,

$$
d X^{0}(t)=b(t) d t+\sigma(t) d B(t)+\int_{\mathbb{R}_{0}} \theta(t, z) \tilde{N}(d t, d z) ; t \in[0, T] .
$$

We consider the optimal singular control problem

$$
\sup _{\xi \in \mathcal{A}_{\mathcal{E}}} J(\xi)
$$

where $J(\xi)$ is as in (2.3), that is,

$$
J(\xi)=E\left[\int_{0}^{T} f\left(t, X^{\xi}(t), \omega\right) d t+g\left(X^{\xi}(T), \omega\right)+\int_{0}^{T} h\left(t, X^{\xi}\left(t^{-}\right), \omega\right) d \xi(t)\right]
$$

with the additional assumptions that $f$ and $g$ are $C^{2}$ with respect to $x$,

$$
g^{\prime \prime}(x) \leq 0, \quad \frac{\partial^{2} f}{\partial x^{2}}(s, x) \leq 0, \quad \text { and } \frac{\partial h}{\partial x}(s, x) \geq 0 \text { for all } s, x,
$$

and at least one of these three inequalities is strict for all $s, x$. In the following, we set

$$
\tilde{h}(t, x)=\frac{h(t, x)}{-\lambda(t)}
$$

Copyright $@$ by SIAM. Unauthorized reproduction of this article is prohibited. 
We now prove a key lemma which allows us to provide connections between optimality conditions for Problem (2.74) and reflected BSDEs in the next section.

Lemma 2.10. Let $X^{\xi}(t)$ be the state process (2.72) when a control $\xi$ is applied and $X^{0}(t)$ be the uncontrolled state process (2.73). We have the equality

$$
\begin{array}{r}
E\left[g^{\prime}\left(X^{\xi}(T)\right)+\int_{t}^{T} \frac{\partial f}{\partial x}\left(s, X^{\xi}(s)\right) d s+\int_{t^{+}}^{T} \frac{\partial h}{\partial x}\left(s, X^{\xi}\left(s^{-}\right)\right) d \xi(s)-\tilde{h}\left(t, X^{\xi}(t)\right) \mid \mathcal{E}_{t}\right] \\
=E\left[g^{\prime}\left(X^{0}(T)\right)+\int_{t}^{T} \frac{\partial f}{\partial x}\left(s, X^{0}(s)\right) d s+K_{T}^{\xi}-K_{t}^{\xi}-\Lambda_{t}^{\xi} \mid \mathcal{E}_{t}\right]
\end{array}
$$

where

$$
K_{t}^{\xi}=\int_{0}^{t} \gamma^{\xi}(u) d \xi(u)
$$

with

$$
\begin{array}{r}
\gamma^{\xi}(u)=E\left[\left(g^{\prime \prime}\left(X^{0}(T)+\int_{0}^{u} \lambda(s) d \xi(s)\right)+\int_{u}^{T} \frac{\partial^{2} f}{\partial x^{2}}\left(s, X^{0}(s)\right.\right.\right. \\
\left.\left.\left.+\int_{0}^{u} \lambda(r) d \xi(r)\right) d s\right) \lambda(u)+\frac{\partial h}{\partial x}\left(u, X^{\xi}(u)\right) \mid \mathcal{E}_{u}\right]
\end{array}
$$

and

$$
\begin{aligned}
& \Lambda_{t}^{\xi}=E\left[\tilde{h}\left(t, X^{\xi}(t)\right)-\int_{0}^{t}\left(g^{\prime \prime}\left(X^{0}(T)+\int_{0}^{u} \lambda(s) d \xi(s)\right)\right.\right. \\
& \left.\left.\quad+\int_{t}^{T} \frac{\partial^{2} f}{\partial x^{2}}\left(s, X^{0}(s)+\int_{0}^{u} \lambda(r) d \xi(r)\right) d s\right) \lambda(u) d \xi(u) \mid \mathcal{E}_{t}\right] .
\end{aligned}
$$

Proof. We have

$$
\begin{aligned}
g^{\prime}\left(X^{\xi}(T)\right)= & g^{\prime}\left(X^{0}(T)+\int_{0}^{T} \lambda(s) d \xi(s)\right) \\
= & g^{\prime}\left(X^{0}(T)\right)+\int_{0}^{T} g^{\prime \prime}\left(X^{0}(T)+\int_{0}^{u} \lambda(s) d \xi(s)\right) \lambda(u) d \xi(u) \\
= & g^{\prime}\left(X^{0}(T)\right)+\int_{0}^{t} g^{\prime \prime}\left(X^{0}(T)+\int_{0}^{u} \lambda(s) d \xi(s)\right) \lambda(u) d \xi(u) \\
& +\int_{t^{+}}^{T} g^{\prime \prime}\left(X^{0}(T)+\int_{0}^{u} \lambda(s) d \xi(s)\right) \lambda(u) d \xi(u)
\end{aligned}
$$

Copyright (c) by SIAM. Unauthorized reproduction of this article is prohibited. 
and similarly

$$
\begin{aligned}
\int_{t}^{T} & \frac{\partial f}{\partial x}\left(s, X^{\xi}(s)\right) d s \\
= & \int_{t}^{T} \frac{\partial f}{\partial x}\left(s, X^{0}(s)\right) d s \\
& +\int_{t}^{T}\left(\int_{0}^{s} \frac{\partial^{2} f}{\partial x^{2}}\left(s, X^{0}(s)+\int_{0}^{u} \lambda(r) d \xi(r)\right) \lambda(u) d \xi(u)\right) d s \\
= & \int_{t}^{T} \frac{\partial f}{\partial x}\left(s, X^{0}(s)\right) d s \\
& +\int_{0}^{t}\left(\int_{t}^{T} \frac{\partial^{2} f}{\partial x^{2}}\left(s, X^{0}(s)+\int_{0}^{u} \lambda(r) d \xi(r)\right) d s\right) \lambda(u) d \xi(u) \\
& +\int_{t^{+}}^{T}\left(\int_{u}^{T} \frac{\partial^{2} f}{\partial x^{2}}\left(s, X^{0}(s)+\int_{0}^{u} \lambda(r) d \xi(r)\right) d s\right) \lambda(u) d \xi(u)
\end{aligned}
$$

Therefore

$$
\begin{array}{r}
E\left[g^{\prime}\left(X^{\xi}(T)\right)+\int_{t}^{T} \frac{\partial f}{\partial x}\left(s, X^{\xi}(s)\right) d s+\int_{t^{+}}^{T} \frac{\partial h}{\partial x}\left(s, X^{\xi}(s)\right) d \xi(s)-\tilde{h}\left(t, X^{\xi}(t)\right) \mid \mathcal{E}_{t}\right] \\
=E\left[g^{\prime}\left(X^{0}(T)\right)+\int_{t}^{T} \frac{\partial f}{\partial x}\left(s, X^{0}(s)\right) d s+K_{T}^{\xi}-K_{t}^{\xi}-\Lambda_{t}^{\xi} \mid \mathcal{E}_{t}\right],
\end{array}
$$

where $\Lambda_{t}^{\xi}$ is given by (2.81) and

$$
\begin{aligned}
K_{T}^{\xi}-K_{t}^{\xi}:= & \int_{t^{+}}^{T} E\left[g^{\prime \prime}\left(X^{0}(T)+\int_{0}^{u} \lambda(s) d \xi(s)\right)\right. \\
& \left.\left.+\int_{u}^{T} \frac{\partial^{2} f}{\partial x^{2}}\left(s, X^{0}(s)+\int_{0}^{u} \lambda(r) d \xi(r)\right) d s\right) \mid \mathcal{E}_{u}\right] \lambda(u) d \xi(u) \\
+ & \int_{t^{+}}^{T} E\left[\frac{\partial h}{\partial x}\left(u, X^{\xi}(u)\right) \mid \mathcal{E}_{u}\right] d \xi(u) .
\end{aligned}
$$

Thus $K_{t}^{\xi}$ is given by (2.79).

ThEOREm 2.11. Suppose there exists an optimal control $\xi$ for Problem (2.74). Then we have

$$
\begin{array}{r}
E\left[g^{\prime}\left(X^{0}(T)\right)+\int_{t}^{T} \frac{\partial f}{\partial x}\left(s, X^{0}(s)\right) d s+K_{T}^{\xi}-K_{t}^{\xi}-\Lambda_{t}^{\xi} \mid \mathcal{E}_{t}\right] \geq 0 \\
E\left[g^{\prime}\left(X^{0}(T)\right)+\int_{t}^{T} \frac{\partial f}{\partial x}\left(s, X^{0}(s)\right) d s+K_{T}^{\xi}-K_{t}^{\xi}-\Lambda_{t}^{\xi} \mid \mathcal{E}_{t}\right] d K_{t}^{\xi}=0 .
\end{array}
$$

Proof. From Theorem 2.4 and Remark 2.5, we get that the optimality conditions are given by (2.39) which here get the form

$E\left[g^{\prime}\left(X^{\xi}(T)\right)+\int_{t}^{T} \frac{\partial f}{\partial x}\left(s, X^{\xi}(s)\right) d s+\int_{t^{+}}^{T} \frac{\partial h}{\partial x}\left(s, X^{\xi}\left(s^{-}\right)\right) d \xi(s)-\tilde{h}\left(t, X^{\xi}(t)\right) \mid \mathcal{E}_{t}\right] \geq 0$, 


$$
\begin{aligned}
& E\left[g^{\prime}\left(X^{\xi}(T)\right)+\int_{t}^{T} \frac{\partial f}{\partial x}\left(s, X^{\xi}(s)\right) d s\right. \\
& \left.\quad+\int_{t^{+}}^{T} \frac{\partial h}{\partial x}\left(s, X^{\xi}\left(s^{-}\right)\right) d \xi(s)-\tilde{h}\left(t, X^{\xi}(t)\right) \mid \mathcal{E}_{t}\right] d \xi(t)=0
\end{aligned}
$$

a.s. for all $t \in[0, T]$. Moreover, using (2.76), we see that $K_{t}^{\xi}$ defined by (2.79) is nondecreasing and right-continuous and

$$
d K^{\xi}(t)=0 \Leftrightarrow d \xi(t)=0 \text { for all } \xi \in \mathcal{A}_{\mathcal{E}} .
$$

Using Lemma 2.10, we get that the optimality conditions (2.88)-(2.89) are thus equivalent to $(2.86)-(2.87)$.

3. Connections between optimal singular control, reflected BSDEs, and optimal stopping in partial information. In this section, we provide connections between the singular control problem discussed in subsection 2.5, RBSDEs, and optimal stopping. In the following, we will use the notation $x^{+}=\max (x, 0)$ and $x^{-}=\max (-x, 0) ; x \in \mathbb{R}$.

Definition 3.1 (partial information RBSDEs). Let $F:[0, T] \times \mathbb{R} \times \Omega \rightarrow \mathbb{R}$ be a given function such that $F(t, y, \omega)$ is an $\mathcal{E}_{t}$-adapted process for all $y \in \mathbb{R}$ and $F(\cdot, 0, \cdot) \in L^{2}([0, T] \times \Omega)$. Let $L_{t}$ be a given $\mathcal{E}_{t}$-adapted càdlàg process such that $E\left[\sup _{t \in[0, T]}\left(L_{t}^{+}\right)^{2}\right]<\infty$ and all the jumping times of $L_{t}$ are inaccessible. Let $G \in$ $L^{2}(P)$ be a given $\mathcal{E}_{T}$-measurable random variable such that $G \geq L_{T}$ a.s. We say that a triple $\left(Y_{t}, M_{t}, K_{t}\right)$ is a solution of an RBSDE with driver $F$, terminal value $G$, reflecting barrier $L_{t}$, and partial information filtration $\mathcal{E}_{t} ; t \in[0, T]$ if the following hold:

$$
\begin{aligned}
& Y_{t} \text { is } \mathcal{E}_{t} \text {-adapted and càdlàg, } \\
& M_{t} \text { is an } \mathcal{E}_{t} \text {-martingale and càdlàg, } \\
& E\left[\int_{0}^{T}\left|F\left(s, Y_{s}\right)\right| d s\right]<\infty, \\
& Y_{t}=G+\int_{t}^{T} F\left(s, Y_{s}\right) d s-\left(M_{T}-M_{t}\right)+K_{T}-K_{t}, \quad t \in[0, T], \\
& \text { or equivalently }
\end{aligned}
$$

$$
Y_{t}=E\left[G+\int_{t}^{T} F\left(s, Y_{s}\right) d s+K_{T}-K_{t} \mid \mathcal{E}_{t}\right],
$$

$K_{t}$ is nondecreasing, $\mathcal{E}_{t}$-adapted, and càdlàg, and $K_{0}=0$,

$$
Y_{t} \geq L_{t} \text { a.s. for all } t \in[0, T] \text {, }
$$

$$
\int_{0}^{T}\left(Y_{t}-L_{t}\right) d K_{t}=0 \text { a.s. }
$$

Remark 3.2. The conditions on $L_{t}$ are satisfied if, for example, $L_{t}$ is a Lévy process with finite second moment. See [12]. For conditions which are sufficient to get existence and uniqueness of a solution of the RBSDE, see [11, 12, 13, 18].

3.1. Singular control and RBSDEs in partial information. We now relate the optimality conditions (2.86)-(2.87) for the singular control problem discussed 
in subsection (2.5) - that is, in the special case when (2.71) and (2.76) hold - and RBSDEs.

THEOREM 3.3 (From singular control to RBSDE in partial information). Suppose we can find a singular control $\xi(t)$ such that (2.86)-(2.87) hold. Define

$$
Y_{t}:=E\left[g^{\prime}\left(X^{0}(T)\right)+\int_{t}^{T} \frac{\partial f}{\partial x}\left(s, X^{0}(s)\right) d s+K_{T}^{\xi}-K_{t}^{\xi} \mid \mathcal{E}_{t}\right],
$$

where $K_{t}^{\xi}$ is as in (2.79). Then there exists an $\mathcal{E}_{t}$-martingale $M_{t}$ such that $\left(Y_{t}, M_{t}, K_{t}^{\xi}\right)$ solves the RBSDE (3.1)-(3.8) with

$$
F(t)=E\left[\frac{\partial f}{\partial x}\left(t, X^{0}(t)\right) \mid \mathcal{E}_{t}\right], G=E\left[g^{\prime}\left(X^{0}(T)\right) \mid \mathcal{E}_{T}\right], \quad \text { and } L_{t}=\Lambda_{t}^{\xi},
$$

where $\Lambda_{t}^{\xi}$ is given by $(2.81)$.

Proof. We can write

$$
Y_{t}=E\left[G+\int_{0}^{T} F(s) d s+K_{T}^{\xi} \mid \mathcal{E}_{t}\right]-\int_{0}^{t} F(s) d s-K_{t}^{\xi} .
$$

Define

$$
M_{t}:=E\left[G+\int_{0}^{T} F(s) d s+K_{T}^{\xi} \mid \mathcal{E}_{t}\right]
$$

We get

$$
Y_{t}=-\int_{0}^{t} F(s) d s+M_{t}-K_{t}^{\xi}
$$

In particular, choosing $t=T$,

$$
G=Y_{T}=-\int_{0}^{T} F(s) d s+M_{T}-K_{T}^{\xi} .
$$

Subtracting (3.14) from (3.13) we get

$$
Y_{t}-G=\int_{t}^{T} F(s) d s-\left(M_{T}-M_{t}\right)+K_{T}^{\xi}-K_{t}^{\xi},
$$

which shows that $Y_{t}$ satisfies (3.4). Moreover, the optimality conditions (2.86)-(2.87) can be rewritten $Y_{t} \geq \Lambda_{t}^{\xi}$ and $\left[Y_{t}-\Lambda_{t}^{\xi}\right] d K_{t}^{\xi}=0$.

Next we discuss a converse of Theorem 3.3.

Theorem 3.4 (from RBSDE to singular control in partial information). Set

$$
F(t)=E\left[\frac{\partial f}{\partial x}\left(t, X^{0}(t)\right) \mid \mathcal{E}_{t}\right], G=E\left[g^{\prime}\left(X^{0}(T)\right) \mid \mathcal{E}_{T}\right] .
$$

Suppose there exists a solution $\left(Y_{t}, M_{t}, K_{t}\right)$ of the RBSDE corresponding to $F, G$, and a given barrier $L_{t}$ in the sense of Definition 3.1. Suppose there exists $\hat{\xi}(t)$ such that $K_{t}=K_{t}^{\hat{\xi}}=\int_{0}^{t} \gamma^{\hat{\xi}}(u) d \hat{\xi}(u)$ with $\gamma^{\hat{\xi}}$ given by (2.80) with $\xi=\hat{\xi}$ and $L_{t}=\Lambda^{\hat{\xi}}$ with $\Lambda_{t}^{\xi}$ as 
in (2.81). Then $\hat{\xi}$ is a directional substationary point for the performance $J(\xi)$ given by (2.75) in the sense of Theorem 2.4 with

$$
\begin{gathered}
E\left[\tilde{h}\left(t, X^{\hat{\xi}}(t)\right) \mid \mathcal{E}_{t}\right]=L_{t}+E\left[\int _ { 0 } ^ { t } \left(g^{\prime \prime}\left(X^{0}(T)+\int_{0}^{u} \lambda(s) d \hat{\xi}(s)\right)+\int_{t}^{T} \frac{\partial^{2} f}{\partial x^{2}}\left(s, X^{0}(s)\right.\right.\right. \\
\left.\left.\left.+\int_{0}^{u} \lambda(r) d \hat{\xi}(r)\right) d s\right) \lambda(u) d \hat{\xi}(u) \mid \mathcal{E}_{t}\right] .
\end{gathered}
$$

Proof. By Definition 3.1 the process $Y_{t}$ defined as

$$
Y_{t}:=E\left[g^{\prime}\left(X^{0}(T)\right)+\int_{t}^{T} \frac{\partial f}{\partial x}\left(s, X^{0}(s)\right) d s+K_{T}-K_{t} \mid \mathcal{E}_{t}\right], t \in[0, T],
$$

satisfies

$$
Y_{t} \geq L_{t}
$$

and

$$
\left(Y_{t}-L_{t}\right) d K_{t}=0 \text { a.s. } t \in[0, T]
$$

Hence

$$
E\left[g^{\prime}\left(X^{0}(T)\right)+\int_{t}^{T} \frac{\partial f}{\partial x}\left(s, X^{0}(s)\right) d s+K_{T}-K_{t}-L_{t} \mid \mathcal{E}_{t}\right] \geq 0
$$

and

$$
E\left[g^{\prime}\left(X^{0}(T)\right)+\int_{t}^{T} \frac{\partial f}{\partial x}\left(s, X^{0}(s)\right) d s+K_{T}-K_{t}-L_{t} \mid \mathcal{E}_{t}\right] d K_{t}=0, t \in[0, T] .
$$

Suppose there exists a singular control $\hat{\xi}(t)$ such that (2.79)-(2.81) and (3.17) hold. Then, (3.21)-(3.22) coincide with the variational inequalities (2.86)-(2.87) for an optimal singular control $\xi$. These are again equivalent to the variational inequalities (2.30) of Theorem 2.4. Therefore the result follows from Theorem 2.4.

3.2. RBSDEs and optimal stopping in partial information. We first give a connection between reflected BSDEs and optimal stopping problems. The following proposition is an extension to partial information and to the jump case of section 2 in $[10]$.

Proposition 3.5 (reflected partial information BSDEs with jumps and optimal stopping).

Suppose $\left(Y_{t}, M_{t}, K_{t}\right)$ is a solution of the RBSDE (3.1)-(3.8).

(a) Then $Y_{t}$ is the solution of the optimal stopping problem

$$
Y_{t}=\operatorname{ess}_{\tau \in \mathcal{T}_{t, T}^{\mathcal{E}}} E\left[\int_{t}^{\tau} F\left(s, Y_{s}\right) d s+L_{\tau} \chi_{\tau<T}+G \chi_{\tau=T} \mid \mathcal{E}_{t}\right], t \in[0, T],
$$

where $\mathcal{T}_{t, T}^{\mathcal{E}}$ is the set of $\mathcal{E}_{t^{-}}$stopping times $\tau$ with $t \leq \tau \leq T$ and the optimal stopping time is

$$
\begin{aligned}
\hat{\tau}:=\hat{\tau}_{t}: & =\inf \left\{s \in[t, T] ; Y_{s} \leq L_{s}\right\} \wedge T \\
& =\inf \left\{s \in[t, T] ; K_{s}>K_{t}\right\} \wedge T .
\end{aligned}
$$

Copyright (c) by SIAM. Unauthorized reproduction of this article is prohibited. 


$$
K_{T}-K_{T-t}=\max _{s \leq t}\left(G+\int_{T-s}^{T} F\left(r, Y_{r}\right) d r-\left(M_{T}-M_{T-s}\right)-L_{T-s}\right)^{-} ; t \in[0, T] .
$$

Proof. (a) Choose $\tau \in \mathcal{T}_{t, T}^{\mathcal{E}}$. Then by (3.4)

$$
Y_{\tau}=G+\int_{\tau}^{T} F\left(s, Y_{s}\right) d s-\left(M_{T}-M_{\tau}\right)+K_{T}-K_{\tau} .
$$

If we subtract (3.27) from (3.4) and take the conditional expectation we get

$$
\begin{aligned}
Y_{t} & =E\left[\int_{t}^{\tau} F\left(s, Y_{s}\right) d s+Y_{\tau}+K_{\tau}-K_{t} \mid \mathcal{E}_{t}\right] \\
& \geq E\left[\int_{t}^{\tau} F\left(s, Y_{s}\right) d s+L_{\tau} \chi_{\tau<T}+G \chi_{\tau=T} \mid \mathcal{E}_{t}\right] .
\end{aligned}
$$

Since $\tau \in \mathcal{T}_{t, T}^{\mathcal{E}}$ is arbitrary, this proves that

$$
Y_{t} \geq \underset{\tau \in \mathcal{T}_{t, T}^{\mathcal{\varepsilon}}}{\operatorname{ess} \sup _{t} E}\left[\int_{t}^{\tau} F\left(s, Y_{s}\right) d s+L_{\tau} \chi_{\tau<T}+G \chi_{\tau=T} \mid \mathcal{E}_{t}\right] ; t \in[0, T] .
$$

To get equality in (3.29) we define

$$
\hat{\tau}:=\hat{\tau}_{t}:=\inf \left\{s \in[t, T] ; Y_{s} \leq L_{s}\right\} \wedge T .
$$

Then $\hat{\tau}_{t} \in \mathcal{T}_{t, T}^{\mathcal{E}}$ and

$$
\begin{gathered}
E\left[\int_{t}^{\hat{\tau}} F\left(s, Y_{s}\right) d s+L_{\hat{\tau}} \chi_{\hat{\tau}<T}+G \chi_{\hat{\tau}=T} \mid \mathcal{E}_{t}\right] \\
\geq E\left[\int_{t}^{\hat{\tau}} F\left(s, Y_{s}\right) d s+Y_{\hat{\tau}}+K_{\hat{\tau}}-K_{t} \mid \mathcal{E}_{t}\right] .
\end{gathered}
$$

Here we have used that

$$
K_{\hat{\tau}}-K_{t}=0,
$$

which is a consequence of (3.8) and the fact that $K_{t}$ is continuous (see [12]). This completes the proof of (a).

(b) We proceed as in [9], using the Skorohod lemma.

Lemma 3.6 (Skorohod). Let $x(t)$ be a real càdlàg function on $[0, \infty)$ such that $x(0) \geq 0$. Then there exists a unique pair $(y(t), k(t))$ of càdlàg functions on $[0, \infty)$ such that

- $y(t)=x(t)+k(t) ; \quad t \in[0, \infty)$,

- $y(t) \geq 0 ; \quad t \in[0, \infty)$,

- $k(t)$ is nondecreasing and $k(0)=0$,

- $\int_{0}^{\infty} y(t) d k(t)=0$.

Copyright $@$ by SIAM. Unauthorized reproduction of this article is prohibited. 
The function $k(t)$ is given by

$$
k(t)=\max _{s \leq t} x(s)^{-} .
$$

We say that $(y, k)$ is the solution of the Skorohod problem with respect to the given function $x$.

If we compare with Definition 3.1, we see that if we define

$$
\begin{aligned}
y(t) & :=Y_{T-t}-L_{T-t} \\
& =G+\int_{T-t}^{T} F\left(s, Y_{s}\right) d s-\left(M_{T}-M_{T-t}\right)+K_{T}-K_{T-t}-L_{T-t}, \\
x(t) & :=G+\int_{T-t}^{T} F\left(s, Y_{s}\right) d s-\left(M_{T}-M_{T-t}\right)-L_{T-t}, \\
k(t) & :=K_{T}-K_{T-t},
\end{aligned}
$$

then $(y, k)$ solves the Skorohod problem with respect to $x$. Therefore $k(t)$ is characterized by (3.33), i.e., in terms of $K_{t}$ we have

$$
K_{T}-K_{T-t}=\max _{s \leq t}\left(G+\int_{T-s}^{T} F\left(r, Y_{r}\right) d r-\left(M_{T}-M_{T-s}\right)-L_{T-s}\right)^{-}, ; t \in[0, T],
$$

which is (3.26). This completes the proof of Proposition 3.5.

3.3. Optimal singular control and optimal stopping in partial information. We now use the results of the previous sections to find a link between optimal singular control and optimal stopping.

TheOREm 3.7. Suppose we can find an optimal control $\xi \in \mathcal{A}_{\mathcal{E}}$ for the singular control problem of subsection 2.5 and let $X^{0}(t)$ be the uncontrolled state process. Define

$$
Y_{t}=E\left[g^{\prime}\left(X^{0}(T)\right)+\int_{t}^{T} \frac{\partial f}{\partial x}\left(s, X^{0}(s)\right) d s+K_{T}^{\xi}-K_{t}^{\xi} \mid \mathcal{E}_{t}\right],
$$

where $K_{t}^{\xi}$ is defined by (2.79). Then $Y_{t}$ solves the optimal stopping problem

$$
Y_{t}=\underset{\tau \in \mathcal{T}_{t, T}^{\mathcal{\varepsilon}}}{\operatorname{ess} \sup _{t} E}\left[\int_{t}^{\tau} \frac{\partial f}{\partial x}\left(s, X^{0}(s)\right) d s+L_{\tau} \chi_{\tau<T}+g^{\prime}\left(X^{0}(T)\right) \chi_{\tau=T} \mid \mathcal{E}_{t}\right],
$$

where $L_{t}=\Lambda_{t}^{\xi}$ as in (2.81). Moreover, the corresponding optimal stopping time $\hat{\tau}=\hat{\tau}_{t}$ is given by

$$
\begin{aligned}
\hat{\tau}=\hat{\tau}_{t} & =\inf \left\{s \in[t, T] ; Y_{s} \leq L_{s}\right\} \wedge T, \\
& =\inf \left\{s \in[t, T] ; K_{s}^{\xi}>K_{t}^{\xi}\right\} \wedge T, \\
& =\inf \{s \in[t, T] ; \xi(s)>\xi(t)\} \wedge T .
\end{aligned}
$$

Proof. By Theorem 3.3, there exists a càdlàg $\mathcal{E}_{t}$-martingale $M_{t}$ such that $\left(Y_{t}, M_{t}\right.$, $K_{t}^{\xi}$ ) solves the RBSDE (3.1)-(3.8) with $G, F$, and $L$ given by (3.10). Hence from Proposition 3.5, $Y_{t}$ solves the optimal stopping problem (3.38) and the corresponding optimal stopping time $\hat{\tau}=\hat{\tau}_{t}$ is given by (3.39).

Copyright $@$ ㅇ by SIAM. Unauthorized reproduction of this article is prohibited. 
In the following, we use the notation

$$
\frac{\partial^{k} f}{\partial x^{k}}(s, A)=\left.\frac{\partial^{k} f}{\partial x^{k}}(s, x)\right|_{x=A}
$$

for any random variable $A, k=1,2$.

THEOREM 3.8 (from singular control to optimal stopping in partial information). Suppose that for all $x \in \mathbb{R}$ there exists an optimal control $\xi=\xi_{x}(\cdot) \in \mathcal{A}_{\mathcal{E}}$ for the singular control problem of subsection 2.5, that is,

$$
V(x)=\sup _{\xi \in \mathcal{A}_{\mathcal{E}}} J(\xi, x),
$$

where

$$
J(\xi, x)=E\left[\int_{0}^{T} f\left(t, X_{x}^{\xi}(t), \omega\right) d t+g\left(X_{x}^{\xi}(T), \omega\right)+\int_{0}^{T} h\left(t, X_{x}^{\xi}\left(t^{-}\right), \omega\right) d \xi(t)\right]
$$

and

$$
\begin{aligned}
X_{x}^{\xi}(t)= & x+\int_{0}^{t} b(s) d s+\int_{0}^{t} \sigma(s) d B(s)+\int_{0}^{t} \int_{\mathbb{R}_{0}} \theta(s, z) \tilde{N}(d s, d z) \\
& +\int_{0}^{t} \lambda(s) d \xi(s) ; t \in[0, T]
\end{aligned}
$$

Then

$$
V^{\prime}(x)=U(x),
$$

where $U$ is the solution of the partial information optimal stopping problem

$$
U(x)=\sup _{\tau \in \mathcal{T}_{0, T}^{\mathcal{E}}} E\left[\int_{0}^{\tau} \frac{\partial f}{\partial x}\left(s, X_{x}^{0}(s)\right) d s+\hat{h}(\tau, \xi) \chi_{\tau<T}+g^{\prime}\left(X_{x}^{0}(T)\right) \chi_{\tau=T}\right],
$$

where

$$
\begin{aligned}
\hat{h}(\tau, \xi)= & \tilde{h}\left(\tau, X_{x}^{\xi}(\tau)\right) \\
& -E\left[\left\{g^{\prime \prime}\left(X_{x}^{\xi}(\tau)\right)+\int_{\tau}^{T} \frac{\partial^{2} f}{\partial x^{2}}\left(s, X_{x}^{0}(s)+\int_{0}^{\tau} \lambda(r) d \xi(r)\right) d s\right\} \lambda(\tau) \Delta \xi(\tau) \mid \mathcal{E}_{\tau}\right] .
\end{aligned}
$$

Moreover, an optimal stopping time for (3.43) is

$$
\hat{\tau}=\inf \{s \in[0, T] ; \xi(s)>0\} \wedge T .
$$

$$
V^{\prime}(x)=\frac{d}{d x} J(\xi, x)=E\left[g^{\prime}\left(X_{x}^{\xi}(T)\right)+\int_{0}^{T} \frac{\partial f}{\partial x}\left(s, X_{x}^{\xi}(s)\right) d s+\int_{0}^{T} \frac{\partial h}{\partial x}\left(s, X_{x}^{\xi}\left(s^{-}\right)\right) d \xi(s)\right] .
$$

By Lemma 2.10, we have

$$
\begin{aligned}
& E\left[g^{\prime}\left(X_{x}^{\xi}(T)\right)+\int_{0}^{T} \frac{\partial f}{\partial x}\left(s, X_{x}^{\xi}(s)\right) d s+\int_{0^{+}}^{T} \frac{\partial h}{\partial x}\left(s, X_{x}^{\xi}\left(s^{-}\right)\right) d \xi(s)\right] \\
& =E\left[g^{\prime}\left(X_{x}^{0}(T)\right)+\int_{0}^{T} \frac{\partial f}{\partial x}\left(s, X_{x}^{0}(s)\right) d s+K_{T}^{\xi}-K_{0}^{\xi}-\Lambda_{0}^{\xi}+\tilde{h}(0, x)\right] .
\end{aligned}
$$

Copyright (C) by SIAM. Unauthorized reproduction of this article is prohibited. 
Hence, combining (3.45) and (3.46),

$$
\begin{aligned}
V^{\prime}(x)= & E\left[g^{\prime}\left(X_{x}^{0}(T)\right)+\int_{0}^{T} \frac{\partial f}{\partial x}\left(s, X_{x}^{0}(s)\right) d s+K_{T}^{\xi}\right] \\
& -K_{0}^{\xi}-\Lambda_{0}^{\xi}+\tilde{h}(0, x)+\frac{\partial h}{\partial x}(0, x) \Delta \xi(0) .
\end{aligned}
$$

By (2.79)-(2.81), we have

$$
\begin{aligned}
K_{0}^{\xi} & +\Lambda_{0}^{\xi}-\tilde{h}(0, x)-\frac{\partial h}{\partial x}(0, x) \Delta \xi(0) \\
& =\gamma^{\xi}(0) \Delta \xi(0)+\tilde{h}(0, x)-E\left[R^{\xi}(0)\right] \lambda(0) \Delta \xi(0)-\tilde{h}(0, x)-\frac{\partial h}{\partial x}(0, x) \Delta \xi(0) \\
& =E\left[R^{\xi}(0)\right] \lambda(0) \Delta \xi(0)+\frac{\partial h}{\partial x}(0, x) \Delta \xi(0)-E\left[R^{\xi}(0)\right] \lambda(0) \Delta \xi(0)-\frac{\partial h}{\partial x}(0, x) \Delta \xi(0)=0,
\end{aligned}
$$

where

$$
R^{\xi}(0)=g^{\prime \prime}\left(X^{0}(T)+\lambda(0) \Delta \xi(0)\right)+\int_{0}^{T} \frac{\partial^{2} f}{\partial x^{2}}\left(s, X^{0}(s)+\lambda(0) \Delta \xi(0)\right) d s .
$$

Consequently,

$$
V^{\prime}(x)=E\left[g^{\prime}\left(X_{x}^{0}(T)\right)+\int_{0}^{T} \frac{\partial f}{\partial x}\left(s, X_{x}^{0}(s)\right) d s+K_{T}^{\xi}\right]=Y_{0}
$$

with $Y_{0}$ given by (3.37) at $t=0$. Hence, by (3.38),

$$
V^{\prime}(x)=\sup _{\tau \in \mathcal{T}_{0, T}^{\mathcal{\varepsilon}}} E\left[\int_{0}^{\tau} \frac{\partial f}{\partial x}\left(s, X_{x}^{0}(s)\right) d s+\Lambda_{\tau}^{\xi} \chi_{\tau<T}+g^{\prime}\left(X_{x}^{0}(T)\right) \chi_{\tau=T}\right],
$$

where $\Lambda_{t}^{\xi}$ is given by (2.81), i.e.,

$$
\begin{aligned}
\Lambda_{\tau}^{\xi}=E\left[\tilde{h}\left(\tau, X_{x}^{\xi}(\tau)\right)-\int_{0}^{\tau}\left\{g^{\prime \prime}\left(X_{x}^{0}(\tau)+\int_{0}^{u} \lambda(s) d \xi(s)\right)\right.\right. \\
\left.\left.\quad+\int_{\tau}^{T} \frac{\partial^{2} f}{\partial x^{2}}\left(s, X_{x}^{0}(s)+\int_{0}^{u} \lambda(r) d \xi(r)\right) d s\right\} \lambda(u) d \xi(u) \mid \mathcal{E}_{\tau}\right] \\
\geq E\left[\hat{h}(\tau, \xi) \mid \mathcal{E}_{\tau}\right]
\end{aligned}
$$

by (2.76). Therefore

$$
V^{\prime}(x) \geq \sup _{\tau \in \mathcal{T}_{0, T}^{\mathcal{E}}} E\left[\int_{0}^{\tau} \frac{\partial f}{\partial x}\left(s, X_{x}^{0}(s)\right) d s+\hat{h}(\tau, \xi) \chi_{\tau<T}+g^{\prime}\left(X_{x}^{0}(T)\right) \chi_{\tau=T}\right] .
$$

On the other hand, we know by Theorem 3.7 that

$$
\hat{\tau}=\inf \{s \in[0, T] ; \xi(s)>0\} \wedge T
$$

is an optimal stopping time for the optimal stopping problem (3.48). Noting that

$$
\Lambda_{\hat{\tau}}^{\xi}=E\left[\hat{h}(\hat{\tau}, \xi) \mid \mathcal{E}_{\hat{\tau}}\right]
$$

Copyright (c) by SIAM. Unauthorized reproduction of this article is prohibited. 
we therefore get, by (3.48),

$$
\begin{aligned}
V^{\prime}(x) & =E\left[\int_{0}^{\hat{\tau}} \frac{\partial f}{\partial x}\left(s, X_{x}^{0}(s)\right) d s+\hat{h}(\hat{\tau}, \xi) \chi_{\hat{\tau}<T}+g^{\prime}\left(X_{x}^{0}(T)\right) \chi_{\hat{\tau}=T}\right] \\
& \leq \sup _{\tau \in \mathcal{T}_{0, T}^{\mathcal{E}}} E\left[\int_{0}^{\tau} \frac{\partial f}{\partial x}\left(s, X_{x}^{0}(s)\right) d s+\hat{h}(\tau, \xi) \chi_{\tau<T}+g^{\prime}\left(X_{x}^{0}(T)\right) \chi_{\tau=T}\right] .
\end{aligned}
$$

Combining (3.50) and (3.52) we obtain (3.42)-(3.44).

Remark 3.9. In the case of full information $(\mathcal{E}=\mathcal{F})$ and $b=\theta=0, \sigma(t)=$ $1, \lambda(t)=-1$, and $f, g, h$ deterministic, this relation was studied in [14], where a similar result as in Theorem 3.8 was obtained but with $\hat{h}$ replaced by $\tilde{h}=h$. The difference is due to the assumption in [14] that $\xi$ is left-continuous while we assume right-continuity for $\xi$.

Finally we proceed to study the converse of Theorem 3.7, namely, how to get from the solution of a partial information optimal stopping problem to the solution of associated partial information RBSDE and optimal singular control problems, respectively.

To this end, suppose we find the solution process $Y_{t}$ of the partial information optimal stopping problem

$$
Y_{t}:=\underset{\tau \in \mathcal{T}_{t, T}^{\mathcal{E}}}{\operatorname{ess} \sup _{1}} E\left[\int_{t}^{\tau} F\left(s, Y_{s}\right) d s+L_{\tau} \chi_{\tau<T}+G \chi_{\tau=T} \mid \mathcal{E}_{t}\right] ; t \in[0, T],
$$

where $F(s, y)$ is a given $\mathcal{F}_{s}$-adapted càdlàg process for all $y, F(s, y)$ is Lipschitz continuous with respect to $y$, uniformly in $s, E\left[\int_{0}^{T}|F(s, 0)|^{2} d s\right]<\infty, L_{s}$ is a continuous $\mathcal{E}_{s}$-adapted process, and $G \in L^{2}(P)$ is $\mathcal{F}_{T}$-measurable. Define

$$
\phi(t):=\int_{0}^{t} E\left[F(s, Y(s)) \mid \mathcal{E}_{s}\right] d s+\hat{L}_{t} ; t \in[0, T]
$$

where

$$
\hat{L}_{t}:=L_{t} \chi_{t<T}+E\left[G \mid \mathcal{E}_{T}\right] \chi_{t=T}
$$

and consider the Snell envelope $S_{t}$ of $\phi(\cdot)$ defined as

$$
S_{t}=\underset{\tau \in \mathcal{T}_{t, T}^{\mathcal{E}}}{\operatorname{ess} \sup } E\left[\phi(\tau) \mid \mathcal{E}_{t}\right] ; t \in[0, T] .
$$

$S_{t}$ is the smallest $\mathcal{E}_{t}$-supermartingale that dominates $\phi(\cdot)$. See, e.g., [21]. Let

$$
S_{t}=M_{t}-A_{t}
$$

be the Doob-Meyer decomposition of $S$, i.e., $M_{t}$ is an $\mathcal{E}$-martingale and $A_{t}$ is a càdlàg predictable nondecreasing $\mathcal{E}_{t^{-}}$adapted process with $A_{0^{-}}=0$. See, e.g., [19]. Note that

$$
S_{t}=Y_{t}+\int_{0}^{t} E\left[F(s, Y(s)) \mid \mathcal{E}_{s}\right] d s ; \quad t \in[0, T] .
$$

Therefore we get

$$
Y_{t}=-\int_{0}^{t} E\left[F(s, Y(s)) \mid \mathcal{E}_{s}\right] d s+M_{t}-A_{t} ; t \in[0, T] .
$$

Copyright (c) by SIAM. Unauthorized reproduction of this article is prohibited. 
Hence by (3.53) and (3.59)

$$
E\left[G \mid \mathcal{E}_{T}\right]=Y_{T}=-\int_{0}^{T} E\left[F(s, Y(s)) \mid \mathcal{E}_{s}\right] d s+M_{T}-A_{T} .
$$

Subtracting (3.59) from (3.60) we get

$$
Y_{t}=E\left[G \mid \mathcal{E}_{T}\right]+\int_{t}^{T} E\left[F(s, Y(s)) \mid \mathcal{E}_{s}\right] d s-\left(M_{T}-M_{t}\right)+A_{T}-A_{t}
$$

or, equivalently,

$$
Y_{t}=E\left[G+\int_{t}^{T} F(s, Y(s)) d s+A_{T}-A_{t} \mid \mathcal{E}_{t}\right] .
$$

Moreover, since $S_{t}$ dominates $\phi(t)$ we have

$$
Y_{t}=S_{t}-\int_{0}^{t} E\left[F(s, Y(s)) \mid \mathcal{E}_{s}\right] d s \geq \phi(t)-\int_{0}^{t} E\left[F(s, Y(s)) \mid \mathcal{E}_{s}\right] d s,
$$

that is,

$$
Y_{t} \geq \hat{L}_{t}
$$

An important property of the Snell envelope is that $A_{t}$ increases only when $S_{t^{-}}=$ $\phi\left(t^{-}\right)$, i.e., we have (see [13])

$$
\int_{0}^{T}\left(S_{t^{-}}-\phi\left(t^{-}\right)\right) d A_{t}=0
$$

Since $L_{t}$ is continuous, $A_{t}$ is continuous also (see [12]) and we get

$$
\int_{0}^{T}\left(S_{t}-\phi(t)\right) d A_{t}=0
$$

In terms of $Y_{t}$ this gives

$$
\int_{0}^{T}\left(Y_{t}-\hat{L}_{t}\right) d A_{t}=0
$$

Comparing (3.61), (3.62), and (3.64) with Definition 3.1 we get the following conclusion.

Theorem 3.10 (from optimal stopping to RBSDE in partial information). Suppose $Y_{t}$ solves the optimal stopping problem (3.53). Assume that $L_{t}$ is continuous. Let $M_{t}, A_{t}$ be as in (3.57). Then $\left(Y_{t}, M_{t}, A_{t}\right)$ solves the RBSDE of Definition 3.1 with driver $E\left[F(t, Y(t)) \mid \mathcal{E}_{t}\right]$, terminal value $E\left[G \mid \mathcal{E}_{T}\right]$, and barrier $\hat{L}_{t}$ defined in (3.55). Moreover the optimal stopping time for (3.64) is $\hat{\tau}_{t}=\inf \left\{s \in[t, T] ; Y_{s} \leq \hat{L}_{s}\right\} \wedge T=$ $\inf \left\{s \in[t, T] ; A_{s}>A_{t}\right\} \wedge T$.

Combining this result with Theorem 3.4 we get the following.

THEOREM 3.11 (from optimal stopping to singular control in partial information). Suppose $Y_{t}$ solves the optimal stopping problem (3.53). Assume that $L_{t}$ is continuous. Let $A_{t}$ be as in (3.57) and suppose there exists $\hat{\xi} \in \mathcal{A}_{\mathcal{E}}$ such that $A_{t}=K_{t}^{\hat{\xi}}$ and $\hat{L}_{t}=\Lambda_{t}^{\hat{\xi}}$ with $K_{t}, \Lambda_{t}^{\xi}$ defined in (2.79)-(2.81). Then $\hat{\xi}$ is a directional substationary point in the sense of Theorem 2.4 for the performance functional $J(\xi)$ given by (2.3), where we assume that $f, g$, and $h$ can be chosen such that $E\left[F(t, Y(t)) \mid \mathcal{E}_{t}\right]=E\left[\frac{\partial f}{\partial x}\left(t, X^{0}(t)\right) \mid \mathcal{E}_{t}\right], E\left[G \mid \mathcal{E}_{T}\right]=E\left[g^{\prime}\left(X^{0}(T)\right) \mid \mathcal{E}_{T}\right]$, and $\tilde{h}(t, \omega)=\tilde{h}\left(t, X^{\hat{\xi}}(t), \omega\right)$ is given by $(3.17)$.

Copyright $@$ by SIAM. Unauthorized reproduction of this article is prohibited. 
4. Example of monotone follower with partial information. Consider a singularly controlled process $X^{\xi}(t)$ of the form

$$
d X^{\xi}(t)=b(t) d t+\sigma(t) d B(t)+\int_{\mathbb{R}_{0}} \theta(t, z) \tilde{N}(d t, d z)+\lambda(t) d \xi(t) ; X^{\xi}(0)=x \in \mathbb{R},
$$

where $b(t), \sigma(t)$, and $\theta(t, z)$ are given $\mathcal{F}_{t}$-predictable processes and $\lambda(t)<0$ is a given continuous $\mathcal{E}_{t}$-adapted process. The performance functional is assumed to be

$$
J(\xi)=E\left[\int_{0}^{T} f\left(s, X^{\xi}(s)\right) d s+\int_{0}^{T} h(t) d \xi(t)\right],
$$

where $f(t, x)=\alpha(t) x+\frac{1}{2} \beta(t) x^{2}$ and $\alpha, \beta, h$ are given $\mathcal{F}_{t}$-predictable processes; $\beta<$ $0, h<0$. We want to find $\xi^{*} \in \mathcal{A}_{\mathcal{E}}$ and $\Phi \in \mathbb{R}$ such that

$$
\Phi=\sup _{\xi \in \mathcal{A}_{\mathcal{E}}} J(\xi)=J\left(\xi^{*}\right) .
$$

We may regard (4.3) as the problem to keep $X^{\xi}(t)$ as close to 0 as possible by using the control/energy $\xi(t)$, where the cost rate of having the state at the position $x$ is $-f$ and $-h(t)$ is the unit price of the energy $\xi$ at time $t$. The variational inequalities satisfied by an optimal control $\xi^{*}$ for this problem are (see (2.86)-(2.87), (2.79)-(2.81))

$$
\begin{aligned}
& E\left[\int_{t}^{T}\left\{\alpha(s)+\beta(s) X^{0}(s)\right\} d s+K_{T}^{\xi^{*}}-K_{t}^{\xi^{*}}-\Lambda_{t}^{\xi^{*}} \mid \mathcal{E}_{t}\right] \geq 0, \\
& E\left[\int_{t}^{T}\left\{\alpha(s)+\beta(s) X^{0}(s)\right\} d s+K_{T}^{\xi^{*}}-K_{t}^{\xi^{*}}-\Lambda_{t}^{\xi^{*}} \mid \mathcal{E}_{t}\right] d K_{t}^{\xi^{*}}=0,
\end{aligned}
$$

where

$$
\Lambda_{t}^{\xi^{*}}=E\left[-\frac{h(t)}{\lambda(t)} \mid \mathcal{E}_{t}\right]-E\left[\int_{0}^{t}\left(\int_{t}^{T} \beta(s) d s\right) \lambda(u) d \xi^{*}(u) \mid \mathcal{E}_{t}\right]
$$

and

$$
K_{t}^{\xi^{*}}=\int_{0}^{t} E\left[\left(\int_{u}^{T} \beta(s) d s\right) \lambda(u) \mid \mathcal{E}_{u}\right] d \xi^{*}(u) .
$$

We recognize this as a partial information RBSDE of the type discussed in section 3. The solution is to choose $K_{t}^{\xi^{*}}$ to be the downward reflection force (local time) at the barrier $\Lambda_{t}^{\xi^{*}}$ of the process $\tilde{Y}_{t}$ defined by

$$
\tilde{Y}_{t}:=E\left[\int_{t}^{T}\left\{\alpha(s)+\beta(s) X^{0}(s)\right\} d s \mid \mathcal{E}_{t}\right] ; t \in[0, T] .
$$

Thus the solution is to add to $\tilde{Y}_{t}$ exactly the minimum amount $K_{t}^{\xi^{*}}$ needed to make the resulting process $Y_{t}:=\tilde{Y}_{t}+K_{t}^{\xi^{*}}$ stay above $\Lambda_{t}^{\xi^{*}}$ at all times. Assume from now on that

$$
\tilde{Y}_{0}-\Lambda_{0}^{\xi^{*}} \geq 0
$$

Copyright (c) by SIAM. Unauthorized reproduction of this article is prohibited. 
i.e.,

$$
E\left[\int_{0}^{T}\left\{\alpha(s)+\beta(s) X^{0}(s)\right\} d s+\frac{h(0)}{\lambda(0)}+E\left[\left(\int_{0}^{T} \beta(s) d s\right) \lambda(0) \Delta \xi^{*}(0)\right] \geq 0\right.
$$

Using the Skorohod lemma (Lemma 3.6) we therefore get

$$
K_{t}^{\xi^{*}}=\max _{s \leq t}\left(\tilde{Y}_{s}-\Lambda_{s}^{\xi^{*}}\right)^{-} ; 0 \leq t \leq T
$$

In particular, $K_{0}^{\xi^{*}}=0$ and hence $\Delta \xi^{*}(0)=0$. Hence, combining (4.11) with (4.7) we get

$$
\begin{aligned}
\int_{0}^{t} E\left[\left(\int_{u}^{T} \beta(s) d s\right) \lambda(u) \mid \mathcal{E}_{u}\right] d \xi^{*}(u) \\
=\max _{s \leq t}\left(E \left[-\frac{h(s)}{\lambda(s)}-\int_{0}^{s}\left(\int_{s}^{T} \beta(r) d r\right) \lambda(u) d \xi^{*}(u)\right.\right. \\
\left.\left.\quad-\int_{s}^{T}\left\{\alpha(u)+\beta(u) X^{0}(u)\right\} d u \mid \mathcal{E}_{s}\right]^{-}\right) ; 0 \leq t \leq T .
\end{aligned}
$$

Equivalently, in differential form, using $(-x)^{-}=x^{+}$,

$$
\begin{aligned}
& E {\left[\left(\int_{t}^{T} \beta(s) d s\right) \lambda(t) \mid \mathcal{E}_{t}\right] d \xi^{*}(t) } \\
&=d\left(\operatorname { m a x } _ { s \leq t } \left(E \left[\frac{h(s)}{\lambda(s)}+\int_{0}^{s}\left(\int_{s}^{T} \beta(r) d r\right) \lambda(u) d \xi^{*}(u)\right.\right.\right. \\
& \\
&\left.\left.\left.\quad+\int_{s}^{T}\left\{\alpha(u)+\beta(u) X^{0}(u)\right\} d u \mid \mathcal{E}_{s}\right]^{+}\right)\right) ; 0 \leq t \leq T
\end{aligned}
$$

This is a functional stochastic differential equation in the unknown optimal control $\xi^{*}$. Since the equation describes the increment $d \xi^{*}(t)$ as a function of previous values of $\xi^{*}(s) ; s \leq t$, one can in principle use this to determine $\xi^{*}$, at least numerically.

By Theorem 3.7 we conclude that $Y_{t}$ solves the optimal stopping problem

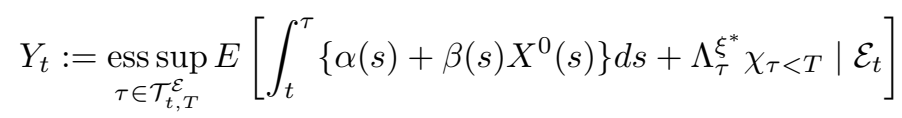

Copyright (c) by SIAM. Unauthorized reproduction of this article is prohibited. 
and the optimal stopping time is

$$
\begin{gathered}
\hat{\tau}_{t}=\inf \left\{s \in[t, T] ; Y_{s} \leq \Lambda_{s}^{\xi^{*}}\right\} \wedge T \\
=\inf \left\{s \in[t, T] ; K_{s}^{\xi^{*}}>K_{t}^{\xi^{*}}\right\} \wedge T \\
=\inf \left\{s \in[t, T] ; \xi^{*}(s)>\xi^{*}(t)\right\} \wedge T \\
=\inf \left\{s \in[t, T] ; \max _{u \leq s}\left(E \left[\frac{h(u)}{\lambda(u)}+\int_{0}^{u}\left(\int_{u}^{T} \beta(r) d r\right) \lambda(y) d \xi^{*}(y)\right.\right.\right. \\
\left.\left.\quad+\int_{u}^{T}\left\{\alpha(r)+\beta(r) X^{0}(r)\right\} d r \mid \mathcal{E}_{u}\right]^{+}\right) \\
>\max _{u \leq t}\left(E \left[\frac{h(u)}{\lambda(u)}+\int_{0}^{u}\left(\int_{u}^{T} \beta(r) d r\right) \lambda(y) d \xi^{*}(y)\right.\right. \\
\left.\left.\left.\quad+\int_{u}^{T}\left\{\alpha(r)+\beta(r) X^{0}(r)\right\} d r \mid \mathcal{E}_{u}\right]^{+}\right)\right\} \wedge T .
\end{gathered}
$$

In particular, if we put $t=0$ we get by (4.15) an explicit formula for the optimal stopping time as follows:

$$
\begin{gathered}
\hat{\tau}_{0}=\inf \left\{s \in[0, T] ; E\left[\frac{h(s)}{\lambda(s)}+\int_{s}^{T}\left\{\alpha(r)+\beta(r) X^{0}(r)\right\} d r \mid \mathcal{E}_{s}\right]^{+}\right. \\
\left.>E\left[\frac{h(0)}{\lambda(0)}+\int_{0}^{T}\left\{\alpha(r)+\beta(r) X^{0}(r)\right\} d r\right]^{+}\right\} \wedge T .
\end{gathered}
$$

We have thus proved the following.

THEOREM 4.1. Suppose that an optimal singular control $\xi^{*}$ for the problem (4.3) exists and that (4.9) holds. Then $\xi^{*}$ satisfies the functional stochastic differential equation (4.13) with initial value $\xi^{*}\left(0^{-}\right)=\xi^{*}(0)=0$. Moreover, the optimal stopping time for the associated optimal stopping problem (4.14) is given by (4.15).

Two simple but still nontrivial special cases follow.

Corollary 4.2. Suppose $\beta(s)=\lambda(s)=h(s)=-1$ and $\alpha(s)=0 ; s \in[0, T]$. Suppose that

$$
E\left[\int_{0}^{T} X^{0}(s) d s\right] \leq 1
$$

Then an optimal singular control $\xi^{*}(t)$ for the problem (4.3) satisfies the functional stochastic differential equation

$$
(T-t) d \xi^{*}(t)=d\left(\max _{s \leq t}\left(1+(T-s) \xi^{*}(s)-E\left[\int_{s}^{T} X^{0}(s) d s \mid \mathcal{E}_{s}\right]\right)^{+}\right)
$$

with initial value $\xi^{*}\left(0^{-}\right)=\xi^{*}(0)=0$. Moreover the optimal stopping expression (4.16) reduces to

$$
\hat{\tau}_{0}=\inf \left\{s \in[0, T] ; E\left[\int_{s}^{T} X^{0}(r) d r \mid \mathcal{E}_{s}\right]<E\left[\int_{0}^{T} X^{0}(r) d r\right]\right\} \wedge T .
$$

Copyright $@$ by SIAM. Unauthorized reproduction of this article is prohibited. 
Proof. Under the given assumptions on the coefficients, assumption (4.17) is easily seen to be equivalent to (4.10).

Corollary 4.3. Suppose that $\mathcal{E}_{t}=\mathcal{F}_{(t-\delta)^{+}} ; t \in[0, T]$ for some constant $\delta>0$ and that $h(t)$ and $\lambda(t)$ are $\mathcal{E}_{t}$-adapted, $\alpha(t)$ and $\beta(t)$ are deterministic, and $b(t)=$ $0 ; t \in[0, T]$. Then the optimal stopping time for the associated optimal stopping time problem is given by

$$
\begin{aligned}
\hat{\tau}_{0}= & \inf \left\{s \in[0, T] ;\left(\frac{h(s)}{\lambda(s)}+\int_{s}^{T}\left\{\alpha(r)+\beta(r) X^{0}\left((s-\delta)^{+}\right)\right\} d r\right)^{+}\right. \\
> & \left.\left(\frac{h(0)}{\lambda(0)}+\int_{0}^{T}\{\alpha(r)+\beta(r) x\} d r\right)^{+}\right\} \wedge T
\end{aligned}
$$

Proof. This follows from (4.16) and the fact that when $b=0, X^{0}(t)$ is a martingale with respect to $\mathcal{F}_{t}$.

Remark 4.4. Even in the special case of Corollary 4.3 the result appears to be new.

Acknowledgments. We thank John J. A. Hosking, Said Hamadène, and MarieClaire Quenez for useful comments.

\section{REFERENCES}

[1] D. Andersson, The relaxed general maximum principle for singular optimal control of diffusions, Systems Control Lett., 58 (2009), pp. 76-82.

[2] S. Bahlali AND B. Mezerdi, A general stochastic maximum principle for singular control problems, Electron. J. Probab., 10 (2005), pp. 988-1004.

[3] S. BAhlali, B. DJehiche, and B. Mezerdi, The relaxed stochastic maximum principles in singular optimal control of diffusions, SIAM J. Control Optim., 46 (2007), pp. 427-444.

[4] S. Bahlali, F. Chighoub, B. Duehiche, and B. Mezerdi, Optimality necessary conditions in singular stochastic control problems with non-smooth coefficients, J. Math. Anal. Appl., 355 (2009), pp. 479-494.

[5] F. M. BAldursson AND I. Karatzas, Irreversible investemnt and industry equilibrium, Finance Stoch., 1 (1997), pp. 69-89.

[6] E. Benth And K. Reikvam, A connection between singular stochastic control and optimal stopping, Appl. Math. Optim., 49 (2004), pp. 27-41.

[7] F. Boetius and M. Kohlmann, Connections between optimal stopping and singular stochastic control, Stochastic Process. Appl., 77 (1998), pp. 253-281.

[8] A. Cadenillas and U. G. Haussmann, The stochastic maximum principle for a singular control problem, Stoch. Stoch. Rep., 49 (1994), pp. 211-237.

[9] M. Chaleyat-Maurel, N. El Karoui, and B. Marchal, Réflexion discontinue et systèmes stochastiques, Ann. Probab., 8 (1980), pp. 1049-1067.

[10] N. El Karoui, C. Kapoudjan, E. Pardoux, S. Peng, and M.-C. Quenez, Reflected solutions of backward SDE's, and related obstacle problems for PDE's, Ann. Probab., 25 (1997), pp. $702-737$.

[11] N. El Karoui, S. Peng, and M.-C. Quenez, BSDEs in finance, Math. Finance, 7 (1997), pp. $1-71$.

[12] S. Hamadène And Y. Ouknine, Backward stochastic differential equations with jumps and random obstacle, Electron. J. Probab., 8 (2003), pp. 1-20.

[13] S. Hamadène And Y. Ouknine, Reflected backward SDEs with general jumps, arXiv:0812.3965v2, 2009.

[14] I. Karatzas and S. E. Shreve, Connections between optimal stopping and singular stochastic control I, Monotone follower problems, SIAM J. Control Optim., 22 (1984), pp. 856-877.

[15] T. Meyer-Brandis, B. Øksendal, And X. Zhou, A mean-field stochastic maximum principle via Malliavin calculus, Stochastics, DOI:10,1080/17442508.2011.6516 19

[16] B. Øksendal And A. Sulem, Applied Stochastic Control of Jump Diffusions, 2nd ed., Springer, New York, 2007.

Copyright $@$ by SIAM. Unauthorized reproduction of this article is prohibited. 
[17] B. ØKSendal ANd A. Sulem, Maximum principles for optimal control of forward-backward stochastic differential equations with jumps, SIAM J. Control Optim., 48 (2009), pp. 29452976.

[18] B. ØKsendal And T. Zhang, Backward Stochastic Differential Equations with Respect to General Filtrations and Applications to Insider Finance, Research report 9, University of Oslo, 2009.

[19] P. Protter, Stochastic Integration and Differential Equations, 2nd ed., Springer, New York, 2004.

[20] R. T. Rockafellar, Convex Analysis, Princeton University Press, Princeton, NJ, 1970.

[21] M. Shashiashvili, Semimartingale inequalities for the Snell envelopes, Stoch. Stoch. Rep., 43 (1993), pp. 65-72.

[22] G. WANG AND Z. WU, The maximum principles for stochastic recursive optimal control problems under partial information, IEEE Trans. Automat. Control, 54 (2009), pp. 1230-1242.

[23] G. WANG AND Z. WU, Kalman-Bucy filtering equations of forward and backward stochastic systems and applications to recursive optimal control problems, J. Math. Anal. Appl., 342 (2008), pp. 1280-1296.

[24] Z. Wu AND F. ZHANG, Stochastic maximum principle for optimal control problems of forwardbackward systems involving impulse controls, IEEE Trans. Automat. Control, 56 (2011), pp. 1401-1406.

[25] J. XIONG AND X. ZHou, Mean-variance portfolio selection under partial information, SIAM J. Control Optim., 46 (2007), pp. 156-175.

Copyright (C) by SIAM. Unauthorized reproduction of this article is prohibited. 